\title{
On the effects of organic matter and sulphur-containing compounds on the CCN activation of combustion particles
}

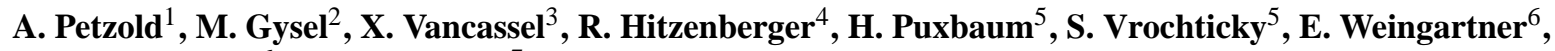 \\ U. Baltensperger ${ }^{6}$, and P. Mirabel ${ }^{7}$ \\ ${ }^{1}$ Institut für Physik der Atmosphäre, DLR Oberpfaffenhofen, 82234 Wessling, Germany \\ ${ }^{2}$ School of Earth, Atmospheric and Environmental Sciences, University of Manchester, P.O. Box 88, Manchester M60 1QD, \\ UK \\ ${ }^{3}$ Atmospheric, Oceanic and Planetary Physics, University of Oxford, Clarendon Laboratory, Parks Road, Oxford OX1 3PU, \\ UK \\ ${ }^{4}$ Institute for Experimental Physics, University of Vienna, Boltzmanngasse 5, 1090 Vienna, Austria \\ ${ }^{5}$ Institute for Chemical Technologies and Analytics, Vienna University of Technology, Getreidemarkt 9/164UPA, 1060 \\ Vienna, Austria \\ ${ }^{6}$ Laboratory of Atmospheric Chemistry, Paul Scherrer Institute (PSI), 5232 Villigen, Switzerland \\ ${ }^{7}$ Université Louis Pasteur, 28, rue Goethe, 67000 Strasbourg, France
}

Received: 1 March 2005 - Published in Atmos. Chem. Phys. Discuss.: 3 May 2005

Revised: 23 August 2005 - Accepted: 10 November 2005 - Published: 1 December 2005

\begin{abstract}
The European PartEmis project (Measurement and prediction of emissions of aerosols and gaseous precursors from gas turbine engines) was focussed on the characterisation and quantification of exhaust emissions from a gas turbine engine. The combustion aerosol characterisation included on-line measurements of mass and number concentration, size distribution, mixing state, thermal stability of internally mixed particles, hygroscopicity, cloud condensation nuclei $(\mathrm{CCN})$ activation potential, and off-line analysis of chemical composition. Based on this extensive data set, the role of sulphuric acid coating and of the organic fraction of the combustion particles for the $\mathrm{CCN}$ activation was investigated. Modelling of $\mathrm{CCN}$ activation was conducted using microphysical and chemical properties obtained from the measurements as input data. Coating the combustion particles with water-soluble sulphuric acid, increases the potential CCN activation, or lowers the activation diameter, respectively. The adaptation of a Köhler model to the experimental data yielded coatings from 0.1 to 3 vol- $\%$ of watersoluble matter, which corresponds to an increase in the fraction of $\mathrm{CCN}$-activated combustion particles from $\leq 10^{-4}$ to $\cong 10^{-2}$ at a water vapour saturation ratio $\mathrm{S}_{w}=1.006$. Additional particle coating by coagulation of combustion particles and aqueous sulphuric acid particles formed by nucleation further reduces the $\mathrm{CCN}$ activation diameter. In contrast, particles containing a large fraction of non-volatile organic com-
\end{abstract}

Correspondence to: A. Petzold

(andreas.petzold@dlr.de) pounds grow significantly less at high relative humidity than particles with a lower content of non-volatile OC. The resulting reduction in the potential $\mathrm{CCN}$ activation with an increasing fraction of non-volatile OC becomes visible as a trend in the experimental data. While a coating of water-soluble sulphuric acid increases the potential CCN activation, or lowers the activation diameter, respectively, the non-volatile organic compounds, mainly found at lower combustion temperatures, can partially compensate this sulphuric acid-related enhancement of CCN activation of carbonaceous combustion aerosol particles.

\section{Introduction}

The indirect aerosol effects are among the most unknown processes in terms of an anthropogenic impact on the global climate. As Lohmann and Feichter (2005) pointed out in a recent review of the global indirect aerosol effects, particularly the role of carbonaceous particles in $\mathrm{CCN}$ activation is unclear. Black carbon which is a major constituent of carbonaceous particles emitted from incomplete combustion processes contributes to the direct aerosol radiative effect by absorption of visible radiation which results in a net reduction in shortwave radiation and thus a negative forcing at the surface. At the top-of atmosphere, carbonaceous particles exert a positive forcing. This effect can be amplified if absorption of solar radiation by carbonaceous particles occurs

(C) 2005 Author(s). This work is licensed under a Creative Commons License. 
within cloud droplets. Since aerosol particles are predominantly a complex internal mixture of chemical substances, the effects of coating insoluble black carbon particles with soluble organic or inorganic species may have a strong effect on the CCN activation of those particles. The knowledge of the coating effects is in turn a prerequisite for an adequate treatment of carbonaceous particles in global climate models.

The effects of surface active and partially soluble organic compounds on the activation of aerosols to $\mathrm{CCN}$ are widely discussed (Saxena et al., 1995; Novakov and Corrigan, 1996; Facchini et al., 1999; Charlson et al., 2001; Broekhuizen et al., 2004), showing that these compounds tend to increase the $\mathrm{CCN}$ activation potential of a particle. Among others, Giebl et al. (2002), Kumar et al. (2003) and Broekhuizen et al. (2004) showed in their studies, that soluble organic acids behave in terms of $\mathrm{CCN}$ activation according to Köhler theory, while insoluble organic acids did not activate at a supersaturation of $0.6 \%$ with dry diamaters up to $140 \mathrm{~nm}$. Broekhuizen et al. (2005) showed in a recent work on the closure between measured and modelled CCN for an anthropogenic aerosol in downtown Toronto that small amounts of soluble matter deposited on an insoluble aerosol have a large effect on the $\mathrm{CCN}$ activation of those particles. They were able to achieve closure between measured and predicted $\mathrm{CCN}$ when using size-resolved chemical information from aerosol mass spectrometer measurements as model input.

The hydration properties of combustion particles were investigated mainly in terms of adsorption/desorption of water molecules (Chughtai et al., 1999; Popovitcheva et al., 2001; Seisel et al., 2004). These studies demonstrate that particles generated from sulphur containing fuels show higher hydration levels than combustion particles generated from sulphur free fuel (Chughtai et al., 1999). CCN activation studies on combustion particles including detailed chemical analysis of organic and inorganic compounds are scarce. Weingartner et al. (1997) investigated the hygroscopic growth behaviour of carbon and diesel soot particles, but without investigating the CCN activation. Lammel and Novakov (1995) investigated the water nucleation properties of carbon and diesel soot particles. They demonstrated that an increasing watersoluble fraction of the particles coincided with an enhanced $\mathrm{CCN}$ activation. However, an experimental study on the interaction of carbonaceous combustion particles and sulphurcontaining species, including particle processing in the exhaust gas, with respect to the $\mathrm{CCN}$ activation process of combustion aerosol is sill missing.

During the European PartEmis project, extensive aerosol, gas and chemi-ion measurements were conducted in the exhaust of a gas turbine combustor under different combustion conditions and fuel sulphur concentrations (Wilson et al., 2004). Data sets on aerosol mass and number concentration, size distribution, mixing state, thermal stability of internally mixed particles, hygroscopicity, cloud condensation nuclei $\mathrm{CCN}$ activation potential and aerosol chemical composition were collected simultaneously from the combustor exhaust (Petzold et al., 2005). The PartEmis results are published in a series of papers focusing on aerosol microphysical properties (Petzold et al., 2003; Nyeki et al., 2004), aerosol hygroscopicity (Gysel et al., 2003), CCN activation (Hitzenberger et al., 2003) and aerosol nucleation (Vancassel et al., 2004). The key findings on emission properties of the combustor are: (1) The emitted aerosol contains primary carbonaceous particles forming inside the combustor, and condensation particles nucleating in the cooling exhaust gas from gaseous precursors. The total carbonaceous fraction (TC) is composed of variable fractions of organic (OC) and elemental (EC) or black (BC) carbon, respectively. The nucleating particles are highly correlated with the sulphuric acid being present in the exhaust gas. (2) When operated at old engine conditions, the combustor emits 0.051 to $0.081 \mathrm{~g} \mathrm{BC}$ per $\mathrm{kg}$ of consumed fuel. Operated at modern engine conditions, emission factors are 0.028 to $0.054 \mathrm{~g} \mathrm{~kg}^{-1}$ which is a factor of 2 lower, while the variation in TC emissions is less pronounced. (3) Mean count median diameters of the combustion particle size distribution are $37-41 \mathrm{~nm}$ for old engine and $39-45 \mathrm{~nm}$ for modern engine conditions. The geometric standard deviation of the size distribution is $1.69(1.66-1.73)$ in both cases. Nucleation mode particles are observed almost exclusively in the size range $D<10 \mathrm{~nm}$. (4) Particles of size $D \geq 30 \mathrm{~nm}$ are almost entirely internally mixed. Particles of sizes $\mathrm{D}<20 \mathrm{~nm}$ contain a considerable volume fraction of compounds that volatilise at $390 \mathrm{~K}(10-15 \%)$ and $573 \mathrm{~K}(4-10 \%)$, while respective fractions decrease to $<5 \%$ for particles of size $\mathrm{D} \geq 50 \mathrm{~nm}$.

Extending the PartEmis focus beyond the investigation of particle formation and processing in aircraft engines, this study investigates the $\mathrm{CCN}$ activation of combustion particles, including the detailed information on particle chemical composition and on particle processing in the exhaust plume. In the presented data analysis, particular emphasis was put on the investigation of the connection between particle chemistry and the formation of cloud droplets on these combustion particles. Since cloud condensation activation of aircraft engine-generated particles is only of minor importance for climate issues, the gas turbine combustor was treated in the presented data analysis simply as a generator for combustion particles and nucleated condensation particles for studies on the interaction of carbonaceous combustion particles and sulphur-containing species during $\mathrm{CCN}$ activation.

Two major scientific questions were addressed: (1) which role play volatile condensation particles forming in the cooling exhaust gas from sulphuric acid for the CCN activation potential of combustion particles? (2) Which role plays the organic fraction of the combustion particles in the $\mathrm{CCN}$ activation potential? Targeted scientific objectives were thus aerosol microphysics and aerosol dynamics of combustion particles, formation of nucleation mode particles from the gas phase, interaction of combustion particles with gaseous and particulate sulphuric acid, speciation of the organic fraction of combustion particles, hygroscopic particle growth 
Table 1. Combustor operating conditions during the PartEmis Combustor experiments.

\begin{tabular}{|c|c|c|}
\hline Combustor Parameters & OLD & MODERN \\
\hline $\mathrm{T}$ combustor inlet, $\mathrm{K}$ & 566 & 766 \\
\hline $\mathrm{T}$ combustor outlet, $\mathrm{K}$ & 1125 & 1448 \\
\hline $\mathrm{P}$ combustor inlet, $10^{5} \mathrm{~Pa}$ & 7.05 & 8.2 \\
\hline Air mass flow, $\mathrm{kg} \mathrm{s}^{-1}$ & 2.12 & 2.12 \\
\hline Fuel flow, $\mathrm{kg} \mathrm{s}^{-1}$ & 0.032 & 0.042 \\
\hline Air fuel ratio & 66 & 50.3 \\
\hline
\end{tabular}

factors at water-subsaturated conditions, and $\mathrm{CCN}$ activation at well-defined water-supersaturated conditions. Modelling of CCN activation of combustion particles was conducted using microphysical and chemical properties obtained from the measurements as input data. Based on this unique data set, the importance of the chemical composition of the organic particle fraction and of the mass transfer of sulphuric acid from the gas phase to the combustion aerosol via condensation deposition and coagulation of nucleated particles and combustion particles was investigated with respect to the resulting CCN activation potential of the emitted carbonaceous combustion aerosol particles.

\section{Experimental methods}

\subsection{Gas turbine combustor and exhaust sampling}

In the framework of PartEmis, a gas turbine combustor was operated at two different operation conditions using fuel with three different fuel sulphur contents ( $\mathrm{FSC}=50,410$, and $\left.1270 \mathrm{mg} \mathrm{kg}^{-1}\right)$. The combustor operation conditions chosen correspond to modern and older engine gas path temperatures at cruise altitude of 35000 feet $(\sim 11700 \mathrm{~m})$. The medium FSC is representative of the contemporary average of aviation fuel. The combustor behaved like a typical aircraft engine combustor with respect to thermodynamic data and main emissions. Table 1 summarises the operation conditions of the combustor.

A specially designed equal area traverse-sampling probe was used to extract exhaust samples from the combustor exit exhaust. The probe provided a radially averaged sample at each circumferential position of the combustor exit nozzle. The upper panel of Fig. 1 shows a front-view of the exhaust nozzle area, looking downstream the flowing gas. The sampling probe was moved stepwise, laterally across the combustor exit (eleven positions), where positions 1 and 11 were close to the combustor walls and position 6 was central. The angles indicate the deviation of the sampling probe from the centre position P6. This approach allowed the lateral distribution of gaseous and aerosol species to be measured. A given operation condition and fuel sulphur content will here-
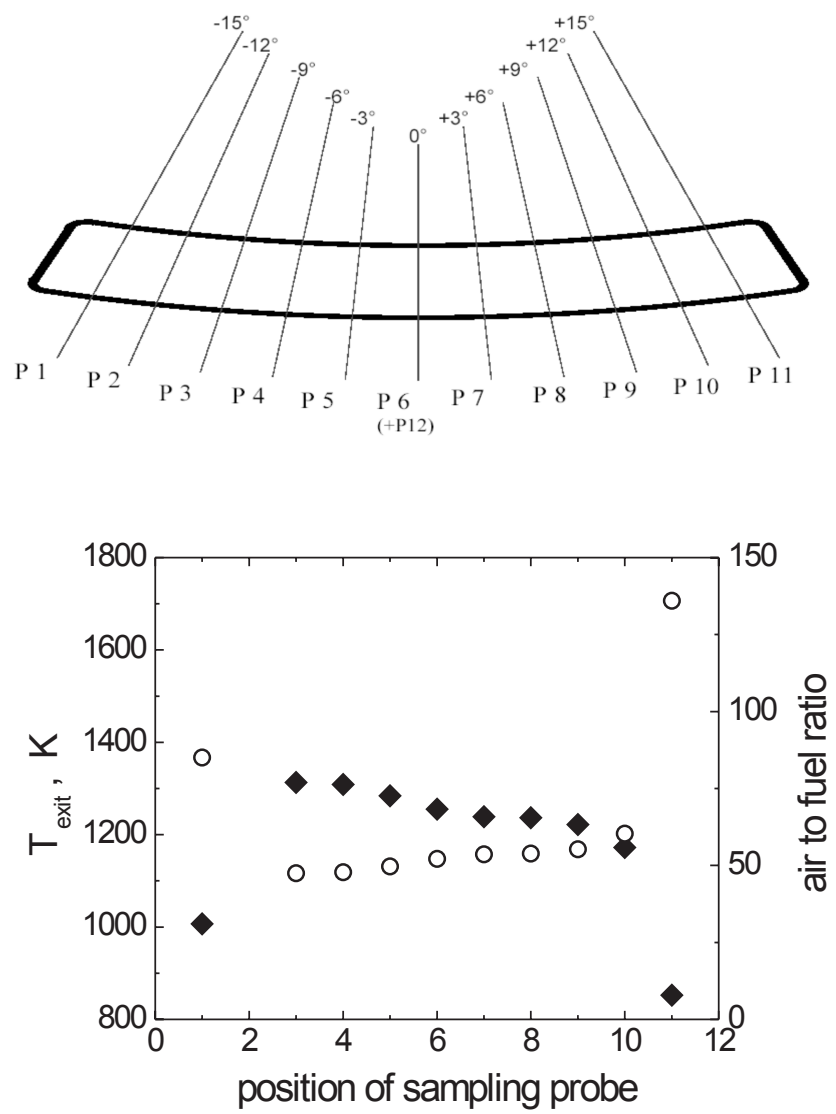

Fig. 1. Combustor exit temperature $\mathrm{T}_{\text {ext }}$ (filled symbols) and ratio of air to fuel (open symbols) for the sampling probe positions on the combustor exit nozzle plane (upper panel) during operation at old cruise conditions.

inafter be referred to as a Combustor test condition, whereas each lateral sampling position will be referred to as a test point. In an aircraft engine gas turbine combustor, dilution air is fed into the combustion chamber downstream the combustion zone through holes in the chamber wall. As shown in Fig. 1 (lower panel), the air to fuel ratio was distinctly higher and the combustor exit temperature $T_{\text {exit }}$ was significantly lower at the edge positions of the probe close to the chamber walls. This feature allowed for additional investigations of the influence of the combustion temperature on particle composition and $\mathrm{CCN}$ activation potential. The sampling probe was kept at each position for at least $20 \mathrm{~min}$ to allow for a sufficient sampling volume for each measurement method.

The sample was cooled to $\sim 150^{\circ} \mathrm{C}$ within the water cooled sampling probe and delivered to undiluted and diluted sample lines. The undiluted line was insulated along its entire length in order to keep the temperature at $\sim 150^{\circ} \mathrm{C}$ to avoid wall losses of combustion products, while the sample in the diluted line naturally attained room temperature $\left(\sim 25^{\circ} \mathrm{C}\right)$ after dilution. Filtered air at room temperature was used for sample dilution, and the dilution factor was determined from 
Table 2. Deployed equipment and measured aerosol properties.

\begin{tabular}{|c|c|}
\hline Aerosol property & Technique \\
\hline \multicolumn{2}{|l|}{ On-line methods } \\
\hline \multirow{3}{*}{$\begin{array}{l}\text { Volatile condensation particles } \\
\text { (number, size) }\end{array}$} & Condensation Particle Size Analyser \\
\hline & (CPSA, Stein et al., 2001; Petzold et al., 2003) \\
\hline & Differential Mobility Analyser (DMA) connected to a Thermodenuder \\
\hline \multirow{4}{*}{$\begin{array}{l}\text { Non-volatile carbonaceous particles } \\
\text { (number, size) }\end{array}$} & Condensation Particle Counter (CPC) \\
\hline & Scanning Mobility Particle Sizer (SMPS) \\
\hline & DMA connected to a Thermodenuder \\
\hline & Volatility Tandem-DMA (Nyeki et al., 2004) \\
\hline Aerosol absorption coefficient $\sigma_{a p}$ & Multi-Angle Absorption Photometer (Petzold and Schönlinner, 2004) \\
\hline Water uptake at $R H \leq 100 \%$ & Hygroscopicity Tandem-DMA (Gysel et al., 2002) \\
\hline Cloud condensation nuclei & Thermal gradient CCN counter (Giebl et al., 2002) \\
\hline \multicolumn{2}{|l|}{ Off-line methods } \\
\hline Chemical composition & $\begin{array}{l}\text { Berner low pressure impactor (BLPI) samples; } \\
\text { filter stack samples; } \\
\text { sample analysis via } \\
\text { - gravimetry (total mass) } \\
\text { - multi-step combustion method (organic/elemental carbon) } \\
\text { - evolved gas analysis (volatility of carbonaceous compounds) } \\
\text { - gas chromatography (aliphatic constituents) } \\
\text { - ion chromatography (major ions) }\end{array}$ \\
\hline
\end{tabular}

temperature and pressure corrected $\mathrm{CO}_{2}$ concentrations measured in the supplied dilution air, diluted sample, and undiluted sample (Wilson et al., 2004). The dilution factors during a Combustor test sampling probe traverse varied from 51 to 73 with a standard deviation of less than $10 \%$. Sample line calibration experiments showed that about $20 \%$ by number of combustion particles were lost to the walls of the sample lines. In terms of mass, no significant loss was detectable. Since quantitative analyses of emissions in terms of number and mass are not in the scope of this study, the data were not corrected for loss effects. Furthermore, all on-line methods extracted samples within a length of $3 \mathrm{~m}$ of the diluted sample line with nucleation mode instruments placed at first position, so that all methods faced almost similar aerosol losses. Aerosol losses in the undiluted sampling line do not influence the results of the particle chemical composition, since data are only used in relative numbers referenced to total carbon or total mass. All on-line physical aerosol measurement methods and off-line aerosol chemical analysis methods applied during the PartEmis experiments are summarised in Table 2 .

\subsection{On-line microphysical characterisation}

On-line aerosol instruments were sampling from the diluted sample line, since dilution with filtered air was necessary for the majority of instruments to reduce concentrations below the upper detection limits. Microphysical properties, hygroscopic growth factors and $\mathrm{CCN}$ activation of the emit- ted combustion aerosol were simultaneously measured online. Thus, artefacts from wall effects or sample storage could be excluded. The key sizing instruments were a multichannel condensation particle counter CPSA for particle sizes $\leq 20 \mathrm{~nm}$ and a DMA scanning from 15 to $300 \mathrm{~nm}$, particle volatility and hygroscopicity were determined by respective Tandem DMA systems; see also Table 2. While the condensation particle counters, the DMA, the absorption photometer, and the $\mathrm{CCN}$ counter were measuring continuously during the traverse of the sampling probe across the nozzle exit area, the Tandem DMA instruments measured particle volatility and particle hygroscopic growth at fixed particle size (volatility) or relative humidity (hygroscopic growth), respectively, during one position of the sampling probe. The measurements taken at the different sampling probe positions or test points, respectively, were then merged into one data ensemble for the respective test condition. The particle hygroscopic growth behaviour was determined from this data ensemble. In the case of filter samples, several test points were merged in order to meet the minimum required sample mass. As a consequence, the chemical information from the filter samples is not available for all test points.

The basic aerosol properties used for this study were the number density and particle size distribution of the combustion aerosol (Petzold et al., 2003; Nyeki et al., 2004), the hygroscopic growth factors for defined particle dry sizes and relative humidity (Gysel et al., 2003), and the number of combustion particles activated as $\mathrm{CCN}$ at a saturation ratio 
with respect to water $\mathrm{S}_{w}=1.006-1.007$ (Hitzenberger et al., 2003). The supersaturation in the CCN counter was set to a value close to water cloud supersaturation conditions. The fraction of CCN activated particles, or the activation ratio, respectively, was determined from the ratio of the number density of $\mathrm{CCN}$ and the number density $\mathrm{N}_{20}$ of combustion aerosol particles of size $\mathrm{D}>20 \mathrm{~nm}$ in the sampled gas. The lower cut-off diameter of $20 \mathrm{~nm}$ was chosen in order to focus exclusively on carbonaceous combustion aerosol particles and to exclude potentially nucleated condensation particles with typical diameters $\mathrm{D} \leq 10 \mathrm{~nm}$ from the analysis (Petzold et al., 2003). Furthermore, one channel of the CPSA was set to a $50 \%$ cut-off diameter of $20 \mathrm{~nm}$, so that a continuous monitoring of the number density of the combustion particle mode was possible. The term CCN activation potential is used in the following for the fraction of $\mathrm{CCN}$ activated particles, because this ratio refers to a defined saturation ratio of 1.006-1.007, which is of the same order as saturation ratios met in natural liquid water clouds. When applied to real cloud conditions, the $\mathrm{CCN}$ activation ratio needs to be adapted by modelling to the saturation ratio prevailing in this particular cloud being investigated.

\subsection{Off-line chemical analysis}

Filter stack samplers and low pressure Berner impactors BLPI for total and size-segregated particulate matter chemical analysis were connected to the undiluted sampling line in order to obtain sufficient sampling volumes. The composition of the carbonaceous particle fraction was determined using both multi-step combustion methods for the determination of total carbon (TC) and elemental carbon (EC), and Evolved Gas Analysis methods EGA (Puxbaum, 1979) for measuring thermograms of the thermal stability of carbonaceous compounds. TC was measured with the multi-step combustion method by burning the entire filter sample in an oxygen-rich atmosphere at $1000^{\circ} \mathrm{C}$ and measuring the evolving $\mathrm{CO}_{2}$. EC was determined in a two-step flash-heating procedure based on the thermal method by Cachier et al. (1989). Organic carbon is oxidized for $42 \mathrm{~min}$ at $340^{\circ} \mathrm{C}$ in a pure oxygen atmosphere. The remaining carbon, defined as elemental carbon, is then determined at $650^{\circ} \mathrm{C}(32 \mathrm{~min})$ by a total organic carbon 700 Analyzer (O.I. Analytical). The uncertainty in the determination for the EC fraction of TC as estimated from charring tests (Cachier et al., 1989) is of the order of $15 \%$ for samples which do not contain any natural organic material.

Evolved gas analysis is based on progressive heating of the sample under an oxygen-rich atmosphere. The separation of OC and EC is performed due to the different thermal stabilities of the carbonaceous fractions using a method originally developed by Puxbaum (1979). The samples are placed in a horizontal furnace FROK 200/50/1000 (AHT Austria, Weitersfeld) at room temperature in oxygen and then heated to $800^{\circ} \mathrm{C}$ at a rate of $20 \mathrm{~K} / \mathrm{min}$. The carbon-containing gases

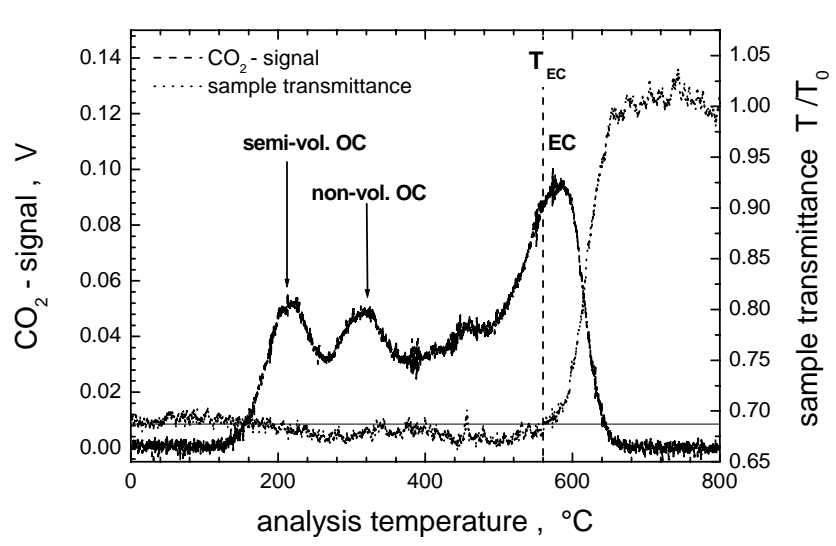

Fig. 2. Example of a "thermogram" including the record of the sample transmittance; the threshold temperature for EC (dashed vertical line) oxidation is reached when the filter transmittance increases above the value of the unheated probe (solid horizontal line).

and vapours evolving from the sample as a result of volatilisation, decomposition, and combustion of the carbonaceous material are converted to $\mathrm{CO}_{2}$ over a $\mathrm{MnO}_{2}$ catalyst maintained at approx. $700^{\circ} \mathrm{C}$ and are detected by a nondispersive infrared analyser (Maihak UNOR 6N). The plot of the rate of evolution of $\mathrm{CO}_{2}$ vs. temperature gives the "thermogram" of carbonaceous material evolved from the sample. A laser beam (diode laser, $\lambda=670 \mathrm{~nm}$ ) monitors the current transmittance of the filter sample, defined as light transmitted through the loaded filter $\mathrm{T}$ vs. light transmitted through a white filter $\mathrm{T}_{0}$. At the start of the EGA, filters loaded with combustion particles are "black", i.e. the transmittance of the sample is low, whereas they are expected to be "white" at the end of the EGA when all collected carbonaceous material is oxidised. In the beginning of the heating process filter transmission slightly decreases because of the charring of the organic material. Filter transmission increases again when elemental carbon is removed from the filter. The OC-EC split is set when the transmittance reaches the same value as in the beginning of the analysis. The carbon evolved after this point is assumed to be elemental carbon. Light scattering effects on the filter transmittance are neglected because filter transmittance is used only as an indicator for the oxidation of lightabsorbing EC. The combined thermo-optical method allows the distinction between $\mathrm{OC}$ and $\mathrm{EC}$ since the oxidisation of organic compounds up to approximately $500^{\circ} \mathrm{C}$ has no or little effect on the filter transmittance, while the oxidation of elemental carbon removes light-absorbing material and increases the filter transmittance. Figure 2 shows an example of a thermogram along with the simultaneously measured sample tramsmittance $\mathrm{T} / \mathrm{T}_{0}$. The EC combustion temperature is at approx. $560^{\circ} \mathrm{C}$ in this case. An estimate of the uncertainty for the EC/TC determination by EGA yielded a value of $5 \%$ when the threshold temperature $\mathrm{T}_{\mathrm{EC}}$ for $\mathrm{EC}$ determination is varied by $\pm 20 \mathrm{~K}$. This uncertainty does not reflect systematic 


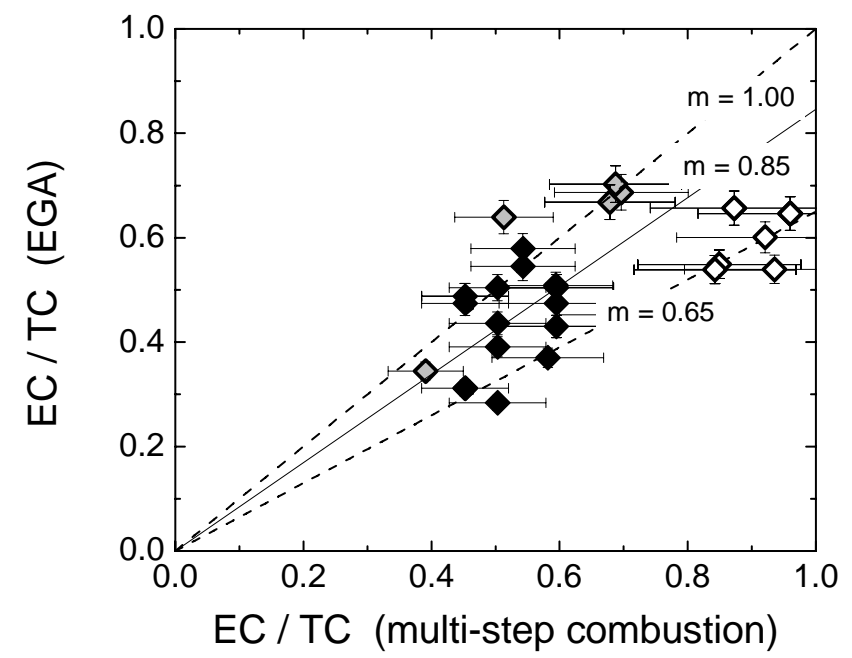

Fig. 3. Correlation of the ratio of elemental carbon to total carbon EC/TC as determined from evolved gas analysis EGA and multistep combustion as a reference method; symbols represent data for low (open), medium (filled) and high (shaded) fuel sulphur content; $\mathrm{m}$ denotes the slope of the linear regression line.

differences in the OC-EC split when compared to other thermal or thermo-optical methods (Schmid et al., 2001).

The different threshold temperatures for the OC-EC separation between multi-step combustion $\left(\mathrm{T}_{\mathrm{EC}}=340^{\circ} \mathrm{C}\right)$ and EGA $\left(\mathrm{T}_{\mathrm{EC}} \cong 560^{\circ} \mathrm{C}\right)$ originate from the different approaches for removing OC by low-temperature oxidation at a constant temperature, or by fast oxidation at higher temperatures. An extensive discussion of the selected temperature threshold for multi-step combustion is given by Cachier et al. (1989). The threshold value $\mathrm{T}_{\mathrm{EC}}$ for $\mathrm{EGA}$ is defined by the filter transmittance signal. In an international aerosol carbon round robin test, multi-step combustion was found to report EC values very close to the average of all deployed methods (Schmid et al., 2001). EGA including the optical correction of charring effects was found to report EC values close to but systematically smaller than values from multi-step combustion. Low-temperature oxidation does not remove as much carbonaceous material from the sample than charring-corrected EGA. Therefore, the remaining EC is higher in the case of multi-step combustion than for EGA. Unfortunately, there exists no commonly agreed method for the thermal determination of OC and EC. All definitions are method-dependent. Keeping this difficulty of EC and OC determination in mind, at least-two thermal methods were deployed in order to get a more reliable OC-EC split for the investigated combustion aerosol.

The definitions for different classes of carbonaceous material referring to the EGA method are: i) semi-volatile $\mathrm{OC}$ (SOC) which evolves for $\mathrm{T}<300^{\circ} \mathrm{C}$, ii) non-volatile $\mathrm{OC}$ (NOC) which evolves for $\mathrm{T}>300^{\circ} \mathrm{C}$ but does generally not influence the filter transmittance, and iii) $\mathrm{EC}$ which evolves

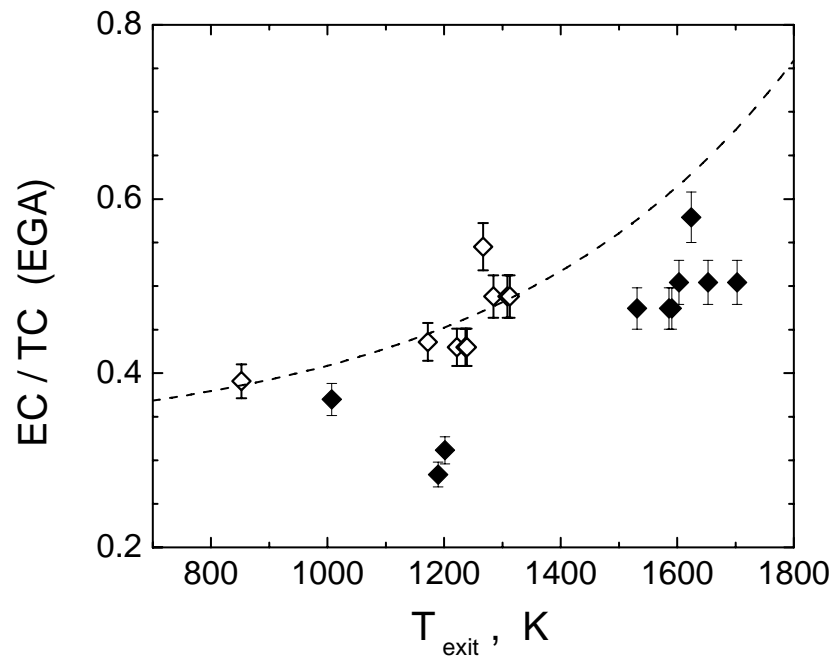

Fig. 4. The elemental fraction of total carbon as a function of the combustor exit temperature for the medium FSC case; open (full) symbols indicate data for old (modern) cruise conditions. Data were determined with EGA, the uncertainty in the ratio EC/TC was determined by varying the analysis temperature $\mathrm{T}_{\mathrm{EC}}$ by $\pm 20 \mathrm{~K}$. The dashed line is inserted as a guideline for the "old cruise condition" cases.

at $\mathrm{T}>500^{\circ} \mathrm{C}$ and increases filter transmittance. The total carbon is $\mathrm{TC}=\mathrm{SOC}+\mathrm{NOC}+\mathrm{EC}$. Black carbon (BC) was determined using Multi-Angle Absorption Photometry (Petzold and Schönlinner, 2004). To determine in particular $\mathrm{SO}_{4}^{2-}$, two Dionex isocratic systems with electrochemical suppression were used.

\section{Results and discussion}

\subsection{Composition of the carbonaceous fraction}

Since the partitioning of carbonaceous compounds between the organic and elemental fractions is essential for this study, the reliability of the determined partitioning values was investigated by the different approaches of EGA and multi-step combustion. As is shown in Fig. 3, the ratio of EC to TC from EGA is approximately $15 \%$ lower than the ratio determined by multi-step combustion. This slight systematic difference between the two applied methods agrees with results from intercomparison studies on carbon methods and is related to different analytical protocols (Schmid et al., 2001). Furthermore, good agreement between EC from multi-step combustion and $\mathrm{BC}$ from aerosol absorption photometry was achieved with an average ratio ( \pm one standard deviation) of $1.05 \pm 0.06$. Thus, EC values as determined by multi-step combustion or by optical means are confirmed, while EGA reports slightly underestimated EC data. However, the slight systematic differences in the OC-EC split will have no influence on the conclusions of this study, since the key property 

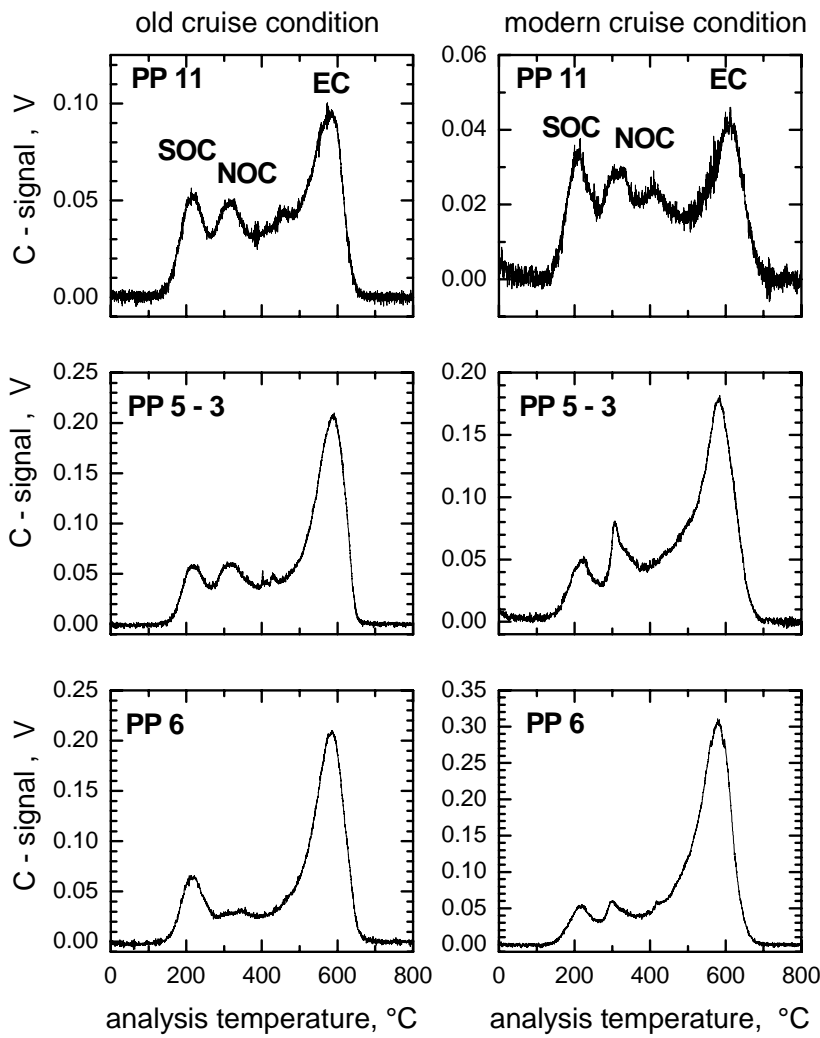

Fig. 5. Thermograms for indicated probe positions for old (left column) and modern (right column) cruise conditions; peaks in the upper two panels are marked as semi-volatile OC (SOC), non-volatile OC (NOC), and EC.

of interest is the split between semi-volatile and non-volatile OC, which can only be determined by EGA. Therefore, all further discussions will refer to the carbonaceous compound classes defined above and determined by EGA.

The EC fraction of TC depends on the exhaust gas temperature and thus on the air to fuel ratio and on the combustion temperature, see Fig. 4. Data were determined with EGA, the uncertainty in the ratio EC/TC was determined by varying the analysis temperature $\mathrm{T}_{\mathrm{EC}}$ by $\pm 20 \mathrm{~K}$. The dashed line is inserted as a guideline for the "old condition" cases. Higher combustion temperatures and thus a more complete combustion result in a higher EC fraction of TC. Exemplary thermograms are compiled in Fig. 5 for old and modern cruise conditions and different sectors of the combustor exit plane. While the EC peak dominates the thermogram for all cases, the intensities of semi- and non-volatile OC peaks vary distinctly with the sampling probe position, most likely caused by varying combustion conditions at the different sections of the combustor where the samples were taken from. Details of the partitioning of carbonaceous compound classes for the medium FSC cases are summarised in Table 3 together with data on the combustor exit temperature, aerosol size distribution, and CCN activation. The medium FSC cases are the

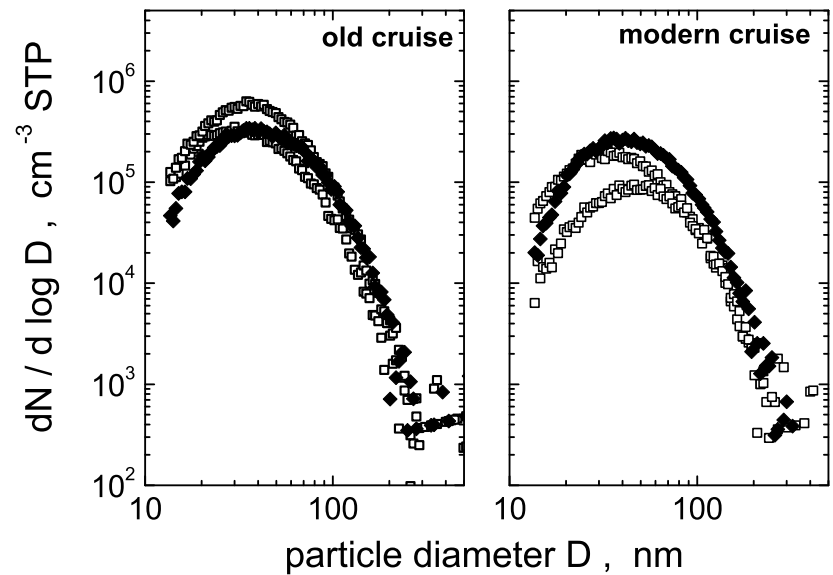

Fig. 6. Particle size distributions measured with an SMPS at edge (open symbols) and centre (filled symbols) positions of the combustor exit area for old and modern cruise conditions; size distributions are corrected for sample dilution.

runs with the highest test point resolution of data for aerosol microphysics, chemical composition and CCN activation.

Figure 6 presents exemplary particle size distributions measured at edge and core positions of the exit plane. The size distributions are corrected for dilution to reflect the number concentration at raw exhaust gas conditions. In contrast to the composition of the carbonaceous fraction, the variation in particle size distribution is less pronounced. However, a weak trend is visible from smaller particles at the edge positions or lower combustor exit temperatures, to larger particles at the centre position at a high combustor exit temperature. The OC-EC partitioning varies in parallel with the exhaust temperature laterally across the combustor exit nozzle test points. Any observed variation of the $\mathrm{CCN}$ activation across the combustor exit is hence expected to be mainly caused by variations of the OC-EC partitioning since the particle size distribution depends only weakly on the sampling probe position.

\subsection{Sulphur-containing aerosol compounds}

During combustion, the sulphur contained in the fuel is oxidised to $\mathrm{SO}_{2}$ which is then partially converted to $\mathrm{S}(\mathrm{VI})$ in the hot exhaust gas. The efficiency of conversion from S(IV) to $\mathrm{S}(\mathrm{VI})$ is described by the conversion efficiency $\varepsilon$ which was on average ( \pm one standard deviation) $2.3 \pm 1.2 \%$ during the PartEmis Combustor experiments (Katragkou et al., 2004). An unknown fraction of the gaseous sulphuric acid may become chemisorbed and linked to the surface of the pre-existing combustion aerosol particles by strong binding forces, or it may condense on these particles during cooling of the exhaust gas. Size-segregated EC and TC values and sulphate content of the combustion particles observed during PartEmis are shown in Fig. 7. The data originate from 
Table 3. Results for the medium FSC experiment ( $\mathrm{FSC}=0.41 \mathrm{~g} \mathrm{~kg}^{-1}$ ) concerning the partitioning of carbonaceous compounds between semivolatile SOC, non-volatile NOC and EC, particle size distribution (CMD=count median diameter, GSD=geometric standard deviation), CCN activation ratio $\mathrm{N}_{\mathrm{CCN}} / \mathrm{N}_{20}$, and activation diameter $\mathrm{D}_{\mathrm{CCN}}$ at $\mathrm{S}_{w}=1.006$ as estimated from the measured size distribution and activation ratio.

\begin{tabular}{|c|c|c|c|c|c|c|c|c|c|}
\hline Condition & Probe Position & $\mathrm{T}_{\text {exit }} \mathrm{K}$ & $\mathrm{SOC} / \mathrm{TC}$ & NOC/TC & $\mathrm{EC} / \mathrm{TC}$ & CMD nm & GSD & $\mathrm{N}_{\mathrm{CCN}} / \mathrm{N}_{20} \times 10^{-3}$ & $\mathrm{D}_{\mathrm{CCN}} \mathrm{nm}$ \\
\hline \multirow[t]{6}{*}{ OLD } & 11 & 852 & 0.17 & 0.44 & 0.39 & 33 & 1.67 & 0.67 & 240 \\
\hline & 10 & 1172 & 0.18 & 0.38 & 0.44 & 38 & 1.62 & 1.24 & 193 \\
\hline & $9-7$ & 1236 & 0.25 & 0.32 & 0.43 & 40 & 1.72 & 1.40 & 192 \\
\hline & 6 & 1267 & 0.14 & 0.32 & 0.54 & & & & \\
\hline & $5-3$ & 1308 & 0.11 & 0.40 & 0.49 & 40 & 1.65 & 1.46 & 199 \\
\hline & 1 & 1007 & 0.32 & 0.31 & 0.37 & 36 & 1.64 & 1.11 & 196 \\
\hline \multirow[t]{5}{*}{ MODERN } & 11 & 1189 & 0.20 & 0.52 & 0.28 & 36 & 1.69 & 2.28 & 182 \\
\hline & $9-7$ & 1652 & 0.10 & 0.40 & 0.50 & 42 & 1.66 & 3.71 & 174 \\
\hline & 6 & 1623 & 0.08 & 0.34 & 0.58 & & & & \\
\hline & $5-3$ & 1585 & 0.11 & 0.41 & 0.48 & 41 & 1.70 & 3.14 & 186 \\
\hline & 1 & 1201 & 0.19 & 0.50 & 0.31 & 47 & 1.73 & 2.76 & 207 \\
\hline
\end{tabular}

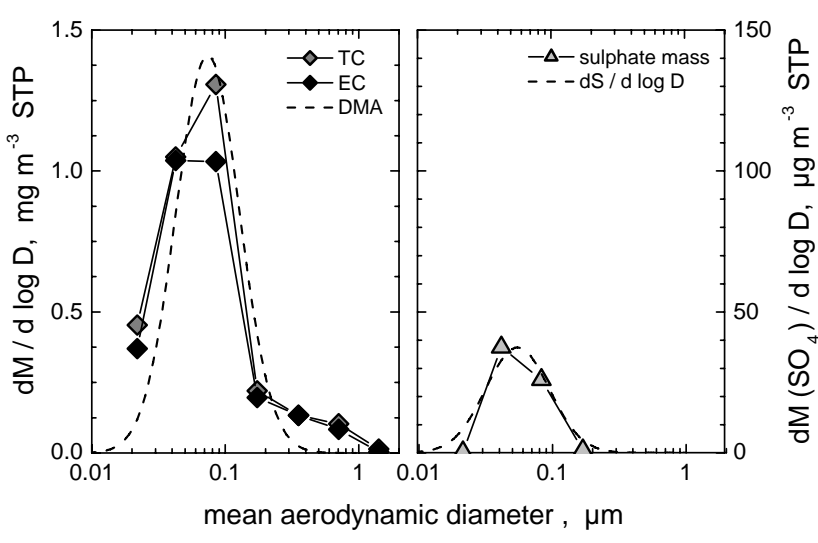

Fig. 7. Size-resolved chemical composition of combustor exhaust particles for high FSC fuel used; left panel shows the mass distribution of total (grey) and elemental (black) carbon from impactor samples and the mass distribution calculated from measured size DMA spectra for spherical particles of unit density $\left(1 \mathrm{~g} \mathrm{~cm}^{-3}\right)$; right panel shows qualitatively the mass distribution of sulphate and a surface distribution calculated from measured DMA spectra.

Berner low pressure impactor samples which were taken at a sample line temperature of $150^{\circ} \mathrm{C}$. At these conditions most of the sulphuric acid will remain in the gas phase, while only a small fraction which is more strongly bound to the particle surface will be observed in particulate matter samples. In Fig. 7, also the mass distribution calculated from DMA spectra with the assumption of spherical particles of unit density is shown in the left panel. The mass distribution of the carbonaceous matter matches the volume distribution of the aerosol quite well. The respective surface distribution is shown in the right panel, but not to scale with the sulphate mass distribution, showing that the mass distribution of sul- phate has its maximum at the same diameter as the surface distribution of the aerosol. Hence, the particulate sulphate at $\mathrm{T} \cong 150^{\circ} \mathrm{C}$ is expected to be bound to the particle surface. The observed particulate sulphate yields a coverage of $\leq 0.1$ monolayers in the high FSC case and even lower values for low and medium FSC cases. This coverage is below the detection limit for all cases when the uncertainties in particle shape and surface area are taken into account.

The coating of carbonaceous combustion particles by reversible condensation of $\mathrm{H}_{2} \mathrm{SO}_{4}$ was determined using Volatility Tandem DMA and Hygroscopicity Tandem DMA methods. Using the V-TDMA, the coating of particles by volatile matter is calculated from a shrinkage factor which describes the reduction in particle size when the volatile matter is vaporised (Nyeki et al., 2004). The H-TDMA method determines the volume fraction of soluble (sulphuric acid) matter from measurements of the hygroscopic growth behaviour as a function of relative humidity (Gysel et al., 2003). It has to be mentioned that both methods measure only particle volatility or particle hygroscopic growth behaviour without specifying the chemical compounds being responsible for these effects. The data can thus only be interpreted as equivalent sulphuric acid coating. As particle volatility and hygroscopic growth are both strongly related to the sulphur content of the burnt fuel and thus to the availability of gaseous sulphuric acid, the coating is assumed to consist of pure sulphuric acid. Both Tandem DMA systems were operated in a closed-loop set-up with sheath air of similar temperature as the aerosol sample, so that evaporation/condensation of sulphuric acid inside the DMA can be excluded. The coating thickness of combustion particles in terms of monolayers of sulphuric acid is plotted in Fig. 8. Both approaches give similar results which show, that only during the runs with high sulphur fuel the coating exceeded a single monolayer 
significantly. For particles in the size range of $100 \mathrm{~nm}$ which defines the upper value of the expected range of activation diameters at the given conditions of $\mathrm{S}_{w}=1.006$, the coating is almost negligible at low FSC and approaches a single monolayer at medium FSC. In the high FSC experiments, the total coverage of particles is about two monolayers of $\mathrm{H}_{2} \mathrm{SO}_{4}$.

Besides the carbonaceous combustion particles, also particles nucleated from gaseous precursors $\mathrm{H}_{2} \mathrm{SO}_{4}$ and $\mathrm{H}_{2} \mathrm{O}$ were detected. These particles occurred preferably in the size range $\mathrm{D}<10 \mathrm{~nm}$ (Petzold et al., 2003). The formation of volatile particles by nucleation was strongly depending on the fuel sulphur content. No particle nucleation was observed in the low and medium fuel sulphur experiments, while strong nucleation was measured during the high sulphur fuel experiments. The right panel of Fig. 9 indicates that predominantly particles in the smallest diameter bin were affected by the fuel sulphur content. Modelling studies using these experimental observations (Vancassel et al., 2004) supported the hypothesis, that the particle nucleation took place downstream the Combustor exit when the sample air was diluted. Additional to the fuel sulphur content, another threshold condition for particle nucleation was the surface area of combustion particles being present in the exhaust gas. The carbonaceous aerosol surface area may act as an additional sink for condensable gases and suppress thus particle nucleation. Figure 9 indeed shows, that particle nucleation was only observed when the surface area was below a threshold of $2300 \mu \mathrm{m}^{2} \mathrm{~cm}^{-3}$.

3.3 CCN activation of sulphuric acid-coated carbonaceous combustion particles

The chemical properties of the carbonaceous and the sulphurcontaining particle fractions including nucleation mode particles were extensively described in the previous subsection. The effects resulting from variations of the organic fraction and the sulphur coating of the carbonaceous combustion particles as well as the potential impact of nucleated $\mathrm{H}_{2} \mathrm{SO}_{4}-$ $\mathrm{H}_{2} \mathrm{O}$ particles on to the $\mathrm{CCN}$ formation potential of the combustion aerosol are discussed in this subsection.

The activation behaviour of combustion particles is described in terms of Köhler theory (Pruppacher and Klett, 1997). The Köhler equation relates the equilibrium gas phase saturation ratio $S_{w}$ with respect to liquid water for a given solution droplet to its chemical composition or water activity $\mathrm{a}_{w}$, respectively, and to the droplet size

$\mathrm{S}_{w}=\mathrm{a}_{w} \times \exp \left[\frac{4 \mathrm{M}_{w} \sigma_{\mathrm{sol}}}{R T \rho_{w} D_{e q}}\right]$.

Water activity $\mathrm{a}_{w}$ and surface tension $\sigma_{\text {sol }}$ of the solution are parameterised as a function of the particles chemical composition (Kulmala et al., 1997). $\mathbf{M}_{w}$ and $\rho_{w}$ are water molecular weight and liquid water density. In the case of combustion aerosol covered by a sulphuric acid layer, the carbonaceous particle core is treated as an insoluble nucleus. The solution

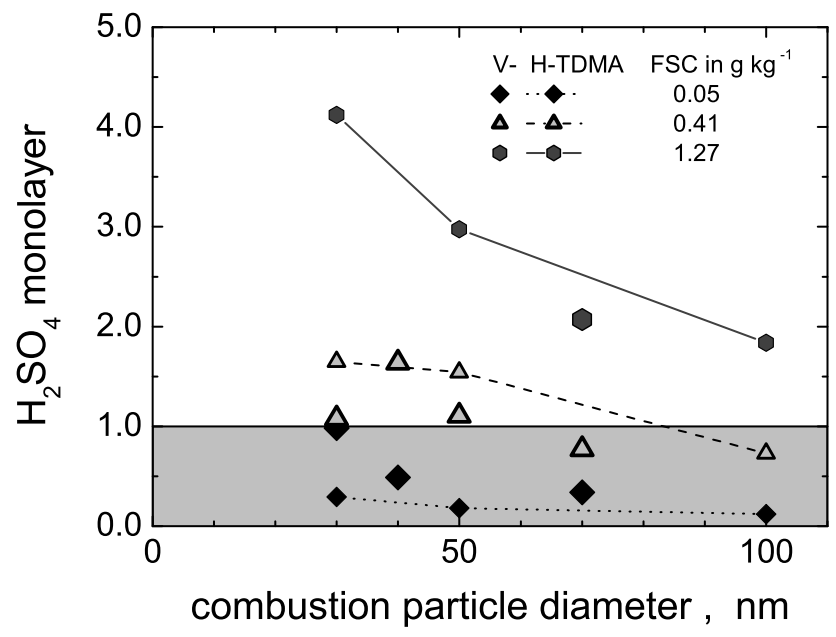

Fig. 8. Coverage of carbonaceous combustion particles in terms of monolayers of $\mathrm{H}_{2} \mathrm{SO}_{4}$ for different levels of fuel sulphur content FSC; the coating was determined from measurements using Volatility Tandem DMA and Hygroscopicity Tandem DMA methods.

forming on the particle at a given saturation ratio $\mathrm{S}_{w}$ is composed of $\mathrm{H}_{2} \mathrm{SO}_{4}$ and $\mathrm{H}_{2} \mathrm{O}$. Depending on the water activity of the solution, the equilibrium water vapour pressure above the droplet may be below water saturation over a flat water surface $\left(S_{w}<1\right)$, or above $\left(S_{w}>1\right)$. The saturation ratio is often expressed as relative humidity $R H=100 \times \mathrm{S}_{w}$ in the subsaturated range. In the absence of soluble matter, the water activity term in the Köhler equation becomes unity, leaving the Kelvin equation which describes the equilibrium $S_{w}$ for a pure water droplet. The Kelvin equation also links $S_{w}$ to the critical size for droplet activation of an insoluble but wettable particle.

Basically two models have been used to calculate activation diameters for a combustion aerosol at a saturation ratio of 1.006 and resulting activation ratios for the measured size distributions. The roughest approximation is the assumption of an insoluble graphite core which undergoes a Kelvin-type activation process. A more appropriate approach is based on a coated-sphere model, where the activation diameter is estimated from measured particle humidity growth factors. This model uses experimental growth factor data and extrapolates the observed growth behaviour up to the desired saturation ratios with respect to water. The only implicit assumption is that the particles consist of an insoluble core and a sulphuric acid coating. Part of the analysis was already published by Gysel et al. (2003) and Hitzenberger et al. (2003), but with consideration of the entire test condition data ensemble only. Details of the interaction between CCN activation and particle chemical composition are subject of the presented analysis.

The growth factor is defined as the ratio between the particle diameter at a certain saturation ratio and the particle 

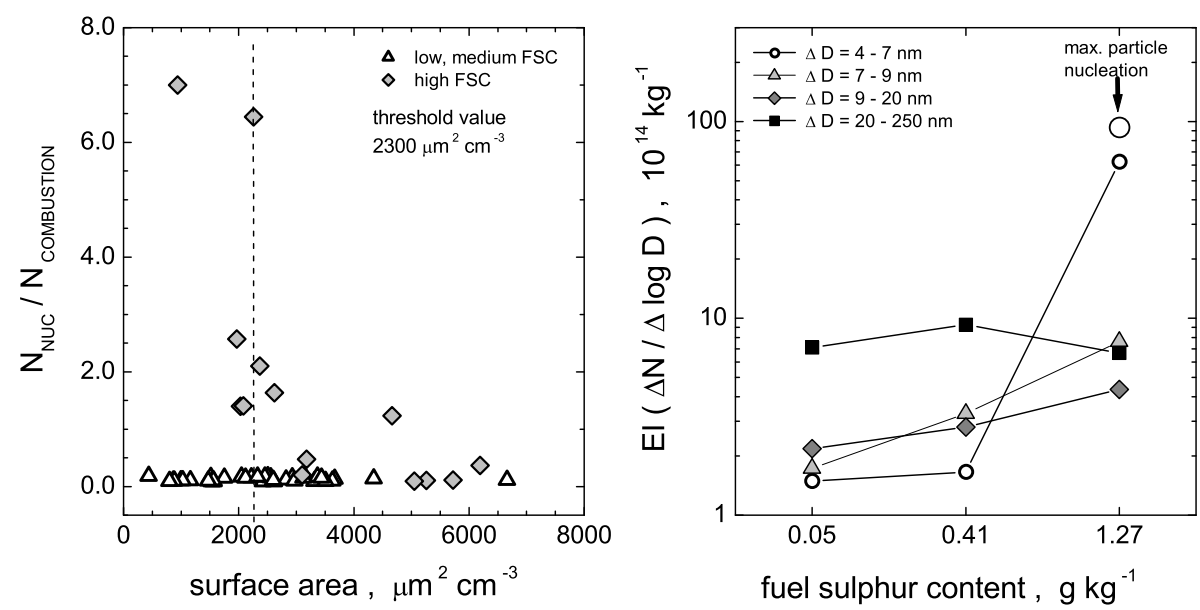

Fig. 9. Left: ratio of nucleated particles $\mathrm{N}_{\mathrm{NUC}}$ of size $\mathrm{D}<10 \mathrm{~nm}$ to combustion particles $\mathrm{N}_{\text {COMBUSTION }}$ of size $\mathrm{D} \geq 20 \mathrm{~nm}$ as a function of the surface area of the pre-existing combustion aerosol; the dashed line denotes the threshold value for particle nucleation of $2300 \mu \mathrm{m}^{2} \mathrm{~cm}^{-3}$. Right: number of particles per $\mathrm{kg}$ of consumed fuel in denoted diameter bins as a function of the fuel sulphur content.

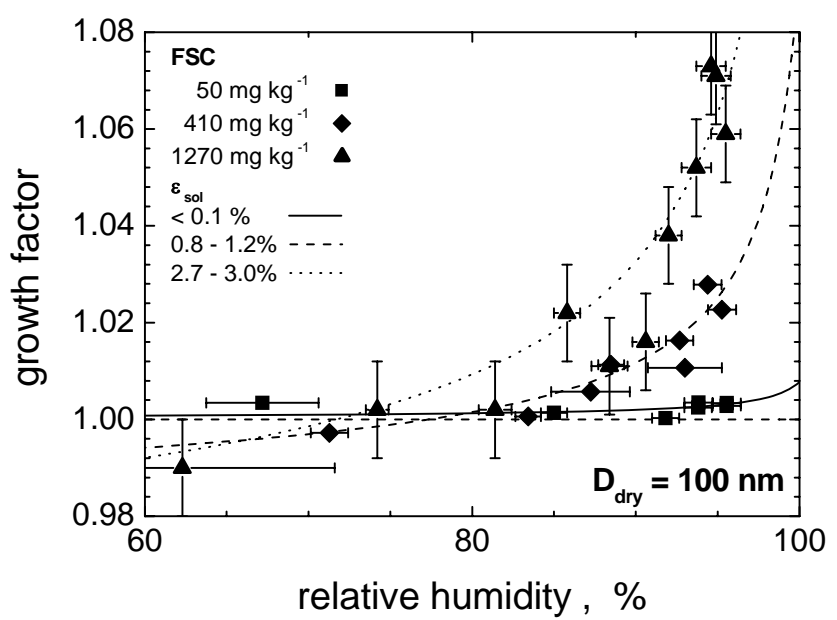

Fig. 10. Humidity growth of particles of initial size $D_{\text {dry }}=100 \mathrm{~nm}$ as a function of relative humidity for low, medium and high fuel sulphur cases; lines represent growth curves determined from Köhler theory for the indicated volume fractions of soluble matter $\varepsilon_{\text {sol }}$.

diameter of the dry particle

$g=\frac{D_{e q}\left(S_{w}\right)}{D_{\mathrm{dry}}}=\frac{D_{e q}(R H)}{D_{\mathrm{dry}}}$

If the particle contains an insoluble core which is coated by a water-soluble layer of volume fraction $\varepsilon_{\text {sol }}$, only the water-soluble material can grow by water uptake (Gysel et al., 2003)

$g\left(\mathrm{~S}_{w}, \varepsilon_{\text {sol }}\right)=\left[1+\varepsilon_{\text {sol }}\left(g_{\text {sol }}^{3}\left(\mathrm{~S}_{w}\right)-1\right)\right]^{\frac{1}{3}}$

$g_{\text {sol }}\left(\mathrm{S}_{w}, \varepsilon_{\text {sol }}\right)$ denotes the theoretical growth factor for a pure soluble particle of equal wet size and can be calculated by Köhler theory.
The experimental particle growth factors were determined using the Hygroscopicity Tandem Differential Mobility Analyser technique H-TDMA (Weingartner et al., 2002; Gysel et al., 2002). Figure 10 shows growth curves for particles of dry size $100 \mathrm{~nm}$, measured with an H-TDMA and modelled with Köhler theory. The volume fraction of watersoluble matter $\varepsilon_{\text {sol }}$ was used as a fitting parameter. The growth behaviour at a given test condition was determined by Köhler curve fitting to the growth factor data set of this specific condition. Figure 10 shows examples for modern conditions and all three investigated FSC cases. The relative humidity was increased from dry to $95 \%$ during the scan of the sampling probe over the combustor exit area. Thus, each data point of the growth curves represents the growth factor of particles with $D_{\text {dry }}=100 \mathrm{~nm}$ as an average over a single position of the sampling probe (test point) during the specified test condition. The obtained volume fractions were $\varepsilon_{\mathrm{sol}}<0.1 \%$ for low FSC, $\varepsilon_{\mathrm{sol}}=0.8-1.2 \%$ for medium FSC and $\varepsilon_{\text {sol }}=2.7-3.0 \%$ for high FSC with lower $\varepsilon_{\text {sol }}$ values referring to "old" and higher $\varepsilon_{\text {sol }}$ values to "modern" operation conditions. Part of the data was already published by Gysel et al. (2003), where also respective values for smaller particles are given. The uncertainty in the growth factor is estimated from Fig. 10: the error bars shown for the high FSC case correspond to an uncertainty in $g$ of \pm 0.01 , which implies that the uncertainty in $g$ is $<0.01$.

Substituting $\mathrm{D}_{e q}$ by $g \times \mathrm{D}_{\text {dry }}$ in Eq. (1) yields the modified Köhler equation

$\mathrm{S}_{w}=a_{w} \times \exp \left[\frac{4 \mathrm{M}_{w} \sigma_{\mathrm{sol}}}{R T \rho_{w} g\left(S_{w}, \varepsilon_{\mathrm{sol}}\right) D_{\mathrm{dry}}}\right]$

From this equation, the equilibrium water vapour pressure can be calculated over particles consisting of an insoluble core and a layer of water-soluble sulphuric acid of volume fraction of $\varepsilon_{\mathrm{sol}}$. 
The upper panel of Fig. 11 shows these so-called Köhler curves for various values of $\varepsilon$ sol and for a given dry size $\mathrm{D}_{\text {dry }}=100 \mathrm{~nm}$ The grey shaded area indicates the saturation ratio with respect to water at which the $\mathrm{CCN}$ counter was operated during PartEmis (Giebl et al., 2002). The drop diameter corresponding to the maximum $\mathrm{S}_{w}$ value, or critical saturation ratio respectively, is the critical diameter which is required for droplet activation at $S_{w}$. The mid panel of Fig. 11 shows the corresponding critical saturation ratios required for the activation of a coated particle of size $D_{\text {dry }}$ for droplet formation, when the same fraction $\varepsilon_{\mathrm{sol}}$ as observed for $D_{\text {dry }}=100 \mathrm{~nm}$ is assumed for all particle diameters $>100 \mathrm{~nm}$. The size dependence of the particle coating was neglected because for the size range $\mathrm{D}>100 \mathrm{~nm}$, no experimental data were available (see Gysel et al., 2003).

For the experimental determination of the activation diameter, the number of droplets forming in the $\mathrm{CCN}$ counter was normalised to the number of combustion particles entering the counter. As explained in Sect. 2.2, the number of particles with diameters $>20 \mathrm{~nm}, \mathrm{~N}_{20}$, was used as the reference number of combustion particles in order to exclude possibly present nucleation mode particles from this analysis. The corresponding number size distributions were integrated from the maximum diameter $0.5 \mu \mathrm{m}$ to smaller sizes until the integrated fraction equalled the activation ratio $\mathrm{N}_{\mathrm{CCN}} / \mathrm{N}_{20}$. The threshold diameter is the measured critical activation diameter $\mathrm{D}_{\mathrm{CCN}}$, i.e.,

$$
\frac{N_{\mathrm{CCN}}}{N_{20}}=\frac{\int_{D_{\mathrm{CCN}}}^{0.5} n(D) d \log D}{\int_{0.02}^{0.5} n(D) d \log D}
$$

$\mathrm{CCN}$ activation diameters determined from Eq. (5) are compiled in Table 3 for the medium FSC cases together with microphysical and chemical data. Similar data sets but with reduced test point resolution are available for the low and high FSC cases. The ensemble CCN activation diameter for each test conditions was calculated from these data sets as average values. The bottom panel of Fig. 11 shows the theoretical dependence of the activation diameter $\mathrm{D}_{\mathrm{CCN}}$ for coated particles on the volume fraction $\varepsilon_{\text {sol }}$ of sulphuric acid, as calculated from Köhler theory for $S_{w}=1.006$. The symbols represent the measured activation diameters as ensemble average values \pm one standard deviation for each test condition. As is demonstrated in the bottom panel of Fig. 11, all average activation diameters determined experimentally for the different fuel sulphur runs of the combustor agree well with the $\mathrm{CCN}$ activation diameters calculated from Köhler theory for a saturation ratio of 1.006 inside the $\mathrm{CCN}$ counter and for insoluble particles coated with sulphuric acid.

Table 4 summarises the activation ratios at $S_{w}=1.006$ and at $\mathrm{S}_{w}=1.01$ calculated for a typical combustion aerosol size distribution as measured in the exhaust of the PartEmis combustor, when Eq. (4) is applied together with the experimental hygroscopic growth data for the determination of $\varepsilon_{\text {sol }}$. Values are compiled for pure insoluble graphite
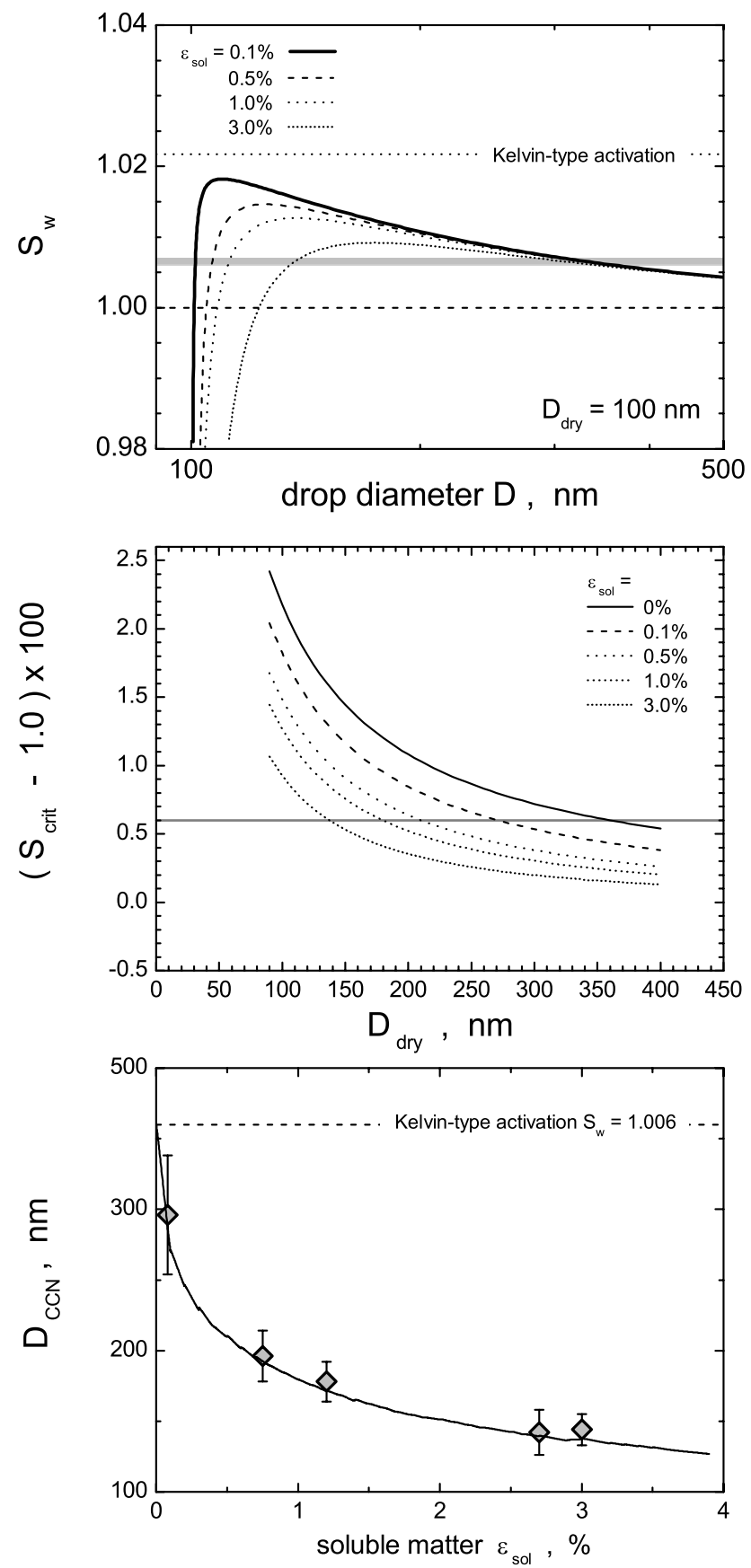

Fig. 11. Top panel: variation of the equilibrium vapour pressure over an aqueous solution drop formed from an insoluble carbonaceous nucleus covered with $\varepsilon_{\text {sol }}$ volume- $\%$ of sulphuric acid in the dry particle state; the grey-shaded area indicates the operation range of the CCN counter. Mid panel: critical saturation ratio for coated particles of dry size $D_{\text {dry }}$. Bottom panel: Theoretical dependence of the activation diameter $\mathrm{D}_{\mathrm{CCN}}$ for insoluble particles coated with $\varepsilon_{\text {sol }}$ volume-\% of sulphuric acid from Köhler theory for $\mathrm{S}_{w}=1.006$, symbols represent the measured activation diameters as ensemble average values \pm one standard deviation for each test condition. 
Table 4. Activation ratios $\mathrm{N}_{\mathrm{CCN}} / \mathrm{N}$ for pure insoluble particles $\left(\varepsilon_{\mathrm{sol}}=0 \%\right)$ and for coated combustion particles $\left(\varepsilon_{\mathrm{sol}}=3 \%\right)$ at a saturation ratio with respect to water of $S_{w}$.

\begin{tabular}{cccc}
\hline $\mathrm{S}_{w}$ & $\varepsilon_{\text {sol }}$ & $\mathrm{D}_{\mathrm{CCN}}, \mathrm{nm}$ & $\mathrm{N}_{\mathrm{CCN}} / \mathrm{N}$ \\
\hline \multirow{2}{*}{1.006} & $0 \%$ & 360 & $\leq 10^{-4}$ \\
& $3 \%$ & 142 & $10^{-3}$ \\
\multirow{2}{*}{1.01} & $0 \%$ & 215 & $3 \times 10^{-4}$ \\
& $3 \%$ & 94 & 0.02 \\
\hline
\end{tabular}

particles without coating $\left(\varepsilon_{\mathrm{sol}}=0 \%\right)$ and for combustion particles coated with 3 vol.-\% sulphuric acid. Data for $\mathrm{S}_{w}=1.01$ are added for comparison with literature data. The coating of graphite particles with sulphuric acid increases the activation ratio for liquid water cloud conditions by more than two orders of magnitude from $\leq 10^{-4}$ to $>10^{-2}$. However, even coated but young combustion particles from gas turbines are still poor CCN.

Before discussing details of the effects of particle coating and organic matter on the $\mathrm{CCN}$ activation potential, the results presented here are compared to experimental data from Lammel and Novakov (1995), who reported activation ratios at a saturation ratio $S_{w}=1.01$ ranging from 1 to $>80 \%$ for different types of combustion particles. The observation of $2 \%$ activated particles with 3 vol.- $\%$ of coating corresponds to the reported activation ratio of $1 \%$ from aviation fuel JP4. A similar value of $1 \%$ of $\mathrm{CCN}$ activated particles emitted from aircraft engines is reported by Pitchford et al. (1991) from in situ measurements. Diesel particles and particles from wood smoke which contain higher fractions of water soluble matter exhibit a much higher $\mathrm{CCN}$ activation with activated fractions of $25-80 \%$. CCN activation diameters reported by Lammel and Novakov (1995) for $\mathrm{S}_{w}=1.005$ are $140 \mathrm{~nm}$ for diesel engine exhaust, which is very close to the values reported in this study, and $60-120 \mathrm{~nm}$ for treated and untreated carbon black particles. Similar to the presented results, the activation diameter decreased with an increasing water-soluble fraction of the particle.

\subsection{Effect of nucleation mode particles on the CCN activa- tion}

For the high FSC experiments, the effect of particle nucleation on the CCN activation of the combustion aerosol was investigated. The underlying question was whether additional transfer of water soluble matter from the gas phase to the surface of the combustion particles by particle nucleation and subsequent coagulation with combustion particles will have a significant effect on the $\mathrm{CCN}$ activation potential of the combustion particles. As having been discussed in Sect. 3.2, particle nucleation was observed only for high FSC cases with the additional condition of a low combustion par-

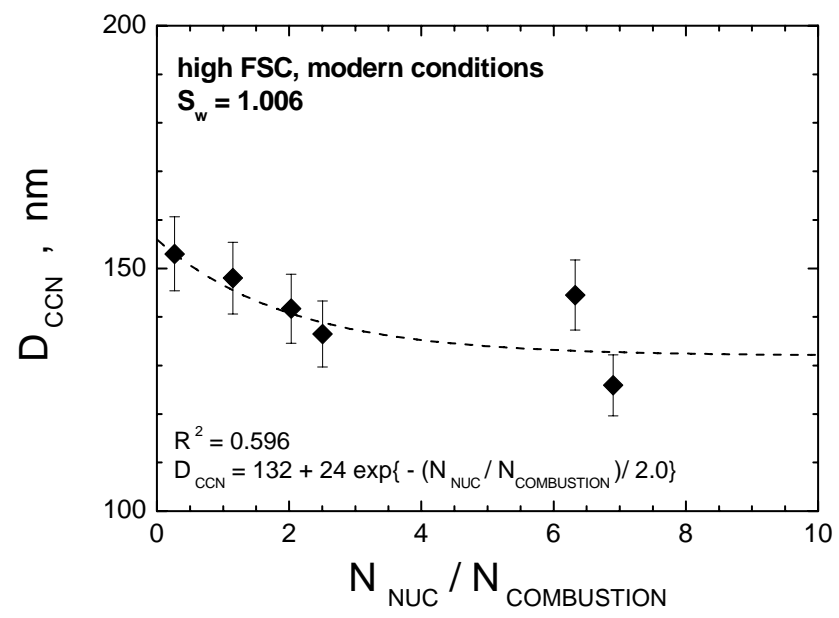

Fig. 12. $C C N$ activation diameter $D_{C C N}$ plotted as a function of the ratio of nucleated particles $\mathrm{N}_{\mathrm{NUC}}$ of size $\mathrm{D}<10 \mathrm{~nm}$ to combustion particles $\mathrm{N}_{\text {COMBUSTION }}$ of size $\mathrm{D} \geq 20 \mathrm{~nm}$ for the high fuel sulphur case and modern operation conditions.

ticle surface area, see also Fig. 9 for details. Test point data are available for high FSC conditions with and without particle nucleation events. During test points for high FSC conditions without particle nucleation the only active $\mathrm{H}_{2} \mathrm{SO}_{4}$ deposition process is gas phase deposition by diffusion. These test points were used as reference cases. In the course of the modelling studies on particle nucleation (Vancassel et al., 2004), the mass transfer of $\mathrm{H}_{2} \mathrm{SO}_{4}$ from the gas phase to the surface of the combustion particles was calculated for test points at high FSC conditions without and with particle nucleation events. It turned out that $60-65 \%$ of the total number of $\mathrm{H}_{2} \mathrm{SO}_{4}$ molecules were deposited on the combustion aerosol surface by deposition from the gas phase, while the remaining $35-40 \%$ was deposited by particle coagulation.

Figure 12 shows the experimentally determined $\mathrm{CCN}$ activation diameters for the high FSC and modern condition runs as a function of the ratio of nucleated particles $\mathrm{N}_{\mathrm{NUC}}$ to combustion particles $\mathrm{N}_{\text {COMBUSTION. The difference between }}$ minimum and maximum values of $\mathrm{D}_{\mathrm{CCN}}$, which correspond to conditions of no and maximum particle nucleation, respectively, is $24 \mathrm{~nm}$. The experimental variability of $\mathrm{D}_{\mathrm{CCN}}$ for a single test condition without particle nucleation is $9-12 \mathrm{~nm}$. Thus, this difference in $\mathrm{D}_{\mathrm{CCN}}$ between no and maximum particle nucleation for high FSC conditions is considered statistically significant. Since the ratio $\mathrm{N}_{\mathrm{NUC}} / \mathrm{N}_{\text {COMBUSTION }}$ is a measure for the strength of particle nucleation, a significant influence of particle nucleation and subsequent coagulation on the CCN activation potential of the combustion aerosol is observed. The activation diameter is lowered from $156 \mathrm{~nm}$ without coagulation mass transfer to $126 \mathrm{~nm}$ at maximum particle nucleation. The reduction in $\mathrm{D}_{\mathrm{CCN}}$ by additional mass transfer of water soluble matter via particle coagulation is of the order of $20 \%$ at $S_{w}=1.006$. The dependence of 


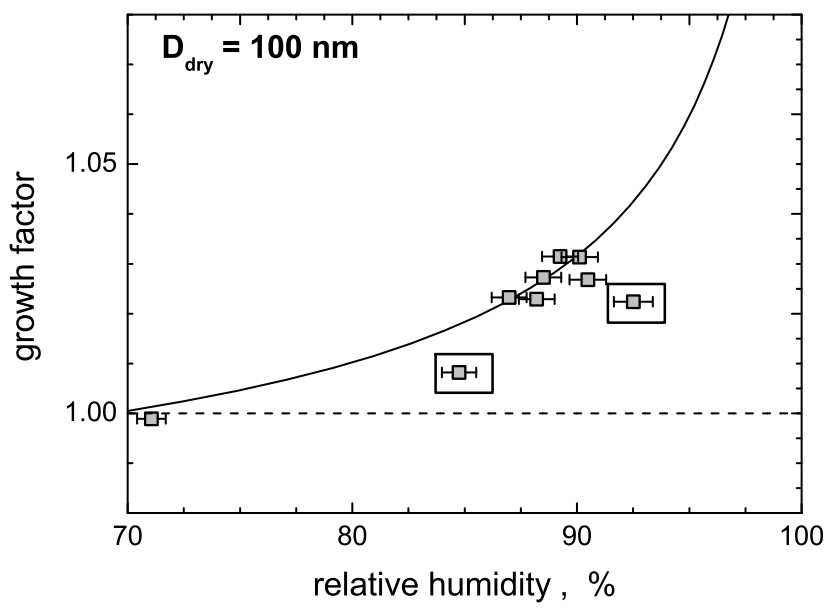

Fig. 13. Humidity growth of particles of initial size $D_{0}=100 \mathrm{~nm}$ as a function of relative humidity for the high fuel sulphur case; the indicated data points correspond to sampling points at the edge positions of the combustor exit nozzle plane.

$\mathrm{D}_{\mathrm{CCN}}$ on the ratio of $\mathrm{N}_{\mathrm{NUC}} / \mathrm{N}_{\text {COMBUSTION }}$ was fitted by an exponential curve yielding the solid line in Fig. 12. The statistical significance is below the confidence level of $95 \%$, so that the plotted curve should be taken as a trend line.

\subsection{CCN activation of combustion particles with a high OC fraction}

The humidity growth factors and resulting $\mathrm{CCN}$ activation diameters for the different experimental parameters, i.e., old and modern conditions and fuel sulphur content levels, were determined from the ensemble of data at the specified test conditions. Each data ensemble consisted of humidity growth factors at different relative humidities which were measured during the scan of the sampling probe across the nozzle exit area, see also Fig. 10 and Sect. 3.3 for details. The scans of the sampling probe included the edge positions, which showed a significant deviation in the OC-fraction of TC compared to the samples from the centre of the nozzle exit plane. Figure 13 demonstrates exemplary for a high FSC case, that not only the OC-TC fraction was different for particles sampled at the edge positions but also the growth behaviour. The growth factors for particles sampled from the edge positions deviate in this case by -0.01 and by -0.019 from the ensemble values, which is significant with respect to an estimated uncertainty in $g$ of $<0.01$. Similar reduced growth factors at the edge positions were observed during all medium and high FSC runs. During low FSC runs, the growth factors were close to unity (Fig. 10) so that possible deviations from the ensemble behaviour were below the resolution of the method.

This observation was a first indicator of a possible influence of the OC fraction of TC on the growth behaviour of combustion particles. In order to investigate whether there

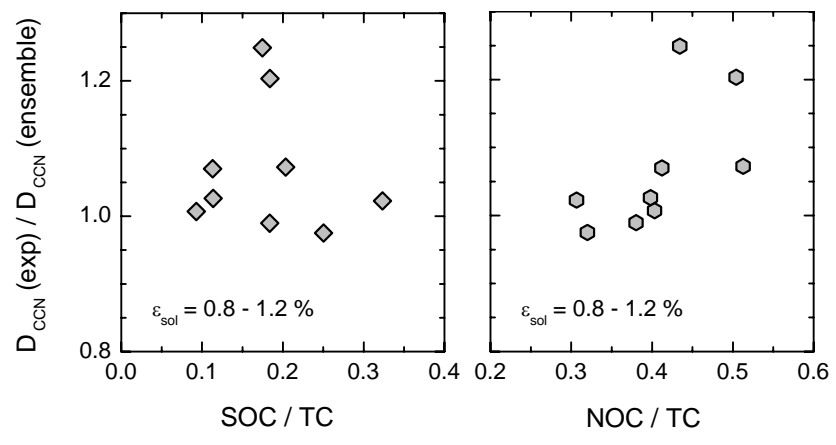

Fig. 14. Ratio of experimentally determined $\mathrm{CCN}$ activation diameters at each investigated test point of medium fuel sulphur content runs to the ensemble $\mathrm{CCN}$ diameter determined from humidity growth factors, as a function of the semi-volatile OC fraction of TC (SOC; left) and the non-volatile OC fraction of TC (NOC; right); the range in $\varepsilon_{\text {sol }}$ corresponds to values for old (lower value) and modern (higher value) combustor operation conditions.

is an impact of the OC fraction of TC on the CCN activation, the activation diameters measured at each sampling probe position for the medium FSC runs were normalised to the ensemble value $\mathrm{D}_{\mathrm{CCN}}$ (ensemble) represented by the Köhler curve fitting all the growth factor measurements at these conditions. The medium FSC runs were selected for this analysis because they are almost complete with respect to chemical, microphysical and $\mathrm{CCN}$ activation data for all sampling probe positions or test points, respectively. The effect of a reduced hygroscopic growth of particle with a high OC fraction was not observed for low FSC cases because the effect was very likely below the resolution of the method. For the high FSC cases, the values of $\mathrm{D}_{\mathrm{CCN}}$ at the edge positions were $175 \mathrm{~nm}$ (old conditions) and $159 \mathrm{~nm}$ (modern conditions), which corresponds to an increase by a factor of 1.25 and 1.16 , respectively, when compared to the ensemble values of 140 and $137 \mathrm{~nm}$ (see Table 5). However, the chemical information on SOC, NOC and EC from thermograms is missing for these test points.

In Fig. 14 , these ratios $\mathrm{D}_{\mathrm{CCN}}(\exp ) / \mathrm{D}_{\mathrm{CCN}}($ ensemble) are plotted as function of the SOC fraction of TC (left) and the NOC fraction of TC (right). An increasing fraction of NOC compounds is correlated to an increase in $\mathrm{D}_{\mathrm{CCN}}$ which in turn reduces the potential of combustion particles for $\mathrm{CCN}$ activation significantly. For SOC no such trend is observable. The maximum increase in $\mathrm{D}_{\mathrm{CCN}}$ with respect to $\mathrm{D}_{\mathrm{CCN}}$ (ensemble) is $\cong 25 \%$ which translates into a reduction of the activation ratio from $2 \times 10^{-3}$ to $9 \times 10^{-4}$ for particles coated with 0.8 vol. $\%$ sulphuric acid. Table 5 summarises the effects of particle coating and organic content on the $\mathrm{CCN}$ activation of combustion particles, based on the observations during PartEmis. CCN activation diameters are given as values averaged over the ensemble of experimental data of one operation condition according to Eq. (5), as theoretical values from ensemble growth curves according to Eq. (4), and as 
Table 5. Activation diameter $\mathrm{D}_{\mathrm{CCN}}$ and activation ratio $\mathrm{N}_{\mathrm{CCN}} / \mathrm{N}$ for the investigated $\mathrm{H}_{2} \mathrm{SO}_{4}-$ coated and non-coated combustion particles for a saturation ratio $S_{w}=1.006$; the stated uncertainties refer to one standard deviation of the respective ensemble average value.

\begin{tabular}{|c|c|c|c|c|}
\hline Test conditions & & Coating $\varepsilon_{\text {sol }}, \%$ & $\begin{array}{l}\text { Activation diameter } \\
\mathrm{D}_{\mathrm{CCN}}, \mathrm{nm}\end{array}$ & Activation ratio $\mathrm{N}_{\mathrm{CCN}} / \mathrm{N}$ \\
\hline insoluble & Kelvin activation & 0.0 & 360 & $10^{-4}$ \\
\hline \multirow[t]{2}{*}{ low FSC, old and modern } & ensemble average value & 0.1 & $290 \pm 43$ & $(8 \pm 2) \times 10^{-4}$ \\
\hline & ensemble growth model & 0.1 & 290 & \\
\hline \multirow[t]{3}{*}{ medium FSC, old } & ensemble average value & 0.8 & $190 \pm 12$ & $(2 \pm 0.6) \times 10^{-3}$ \\
\hline & ensemble growth model & 0.8 & 190 & \\
\hline & NOC fraction $=0.5$ & 0.8 & 237 & $9 \times 10^{-4}$ \\
\hline \multirow[t]{3}{*}{ medium FSC, modern } & ensemble average value & 1.2 & $178 \pm 14$ & $(4 \pm 1) \times 10^{-3}$ \\
\hline & ensemble growth model & 1.2 & 170 & \\
\hline & NOC fraction $=0.5$ & 1.2 & 205 & $3 \times 10^{-3}$ \\
\hline \multirow[t]{3}{*}{ high FSC, old } & ensemble average value & 2.7 & $144 \pm 6$ & $(6 \pm 1) \times 10^{-3}$ \\
\hline & ensemble growth model & 2.7 & 140 & \\
\hline & $\mathrm{N}_{\mathrm{NUC}} / \mathrm{N}_{\text {COMBUSTION }}=2$ & $>2.7$ & 137 & $8 \times 10^{-3}$ \\
\hline \multirow{3}{*}{ high FSC, modern } & ensemble average value & 3.0 & $142 \pm 9$ & $(1 \pm 0.5) \times 10^{-2}$ \\
\hline & ensemble growth model & 3.0 & 137 & \\
\hline & $\mathrm{N}_{\text {NUC }} / \mathrm{N}_{\text {COMBUSTION }}=7$ & $>3.0$ & 132 & $2 \times 10^{-2}$ \\
\hline
\end{tabular}

extreme cases for high OC content (medium FSC runs) and strong particle nucleation (high FSC runs). The respective activation diameter values for the single test points of the medium FSC runs which are plotted in Fig. 14, can be taken from Table 3.

The observed reduction in the hygroscopic growth behaviour of particles sampled at the edge positions of the combustor exit nozzle area may also be explained by a reduced coating with sulphuric acid instead of an influence of the high OC content. The discussion of this hypothesis is based on observations of gas-phase sulphuric acid (Katragkou et al., 2004), particle number concentrations (Petzold et al., 2003) and particle volatility (Nyeki et al., 2004) at the respective probe positions during the different test conditions. The measurements of gaseous sulphuric acid showed that the $\mathrm{H}_{2} \mathrm{SO}_{4}$ mixing ratio was reduced by $25 \%$ at the probe positions closer to the edge of the nozzle exit area compared to the average value over all probe positions sampled during a specific operation condition. However, a similar decrease by $23 \%$ at the edge positions compared to the average value was observed for the aerosol number concentrations of combustion particles, so that the availability of gaseous sulphuric acid per combustion particles for the coating process was almost independent of the sampling probe position. For both $\mathrm{H}_{2} \mathrm{SO}_{4}$ mixing ratio and particle number concentration the reduction is caused by the increased content of dilution air at the edge positions. Furthermore, particles sampled from the edge positions did not show different volatile fractions than particles collected from the centre of the nozzle exit area. Because particle volatile fractions and the availability of gaseous sulphuric acid per combustion particle did not vary significantly between centre and edge positions of the exit nozzle area of the combustor, the observed reduction in the hygroscopic growth can be attributed to the increase in the fraction of non-volatile OC.

As a final assessment of the competing effects of sulphurcontaining compounds and organic matter on the CCN activation of combustion particles, the reduction of the activation diameter with respect to a Kelvin-type activation of insoluble graphite particles is plotted in Fig. 15. Coating the insoluble particle with 0.1 volume- $\%$ of sulphuric acid reduces the activation diameter by $20 \%$ with respect to Kelvin type activation. For a coating of approx. 1.0 and 3.0 vol.- $\%$, respectively, the reduction of $\mathrm{D}_{\mathrm{CCN}}$ is $50 \%$ and $60 \%$. Coating thickness enhancement through coagulation with nucleation mode sulphuric acid - water particles further reduces the $\mathrm{CCN}$ activation diameter to $36 \%$ of the Kelvin-type reference $\mathrm{D}_{\mathrm{CCN}}$. In contrast, a high fraction of nonvolatile organic matter increases the activation diameter by maximum $30 \%$ compared to a similar case with low non-volatile OC content.

\section{Summary and conclusions}

In the course of the European experiment PartEmis the CCN activation of combustion particles generated in a gas turbine combustor was investigated. The presented analysis of the experimental and modelling data showed, that pure carbonaceous particles emitted from a gas turbine combustor are very poor $\mathrm{CCN}$ which behave similar to insoluble particles undergoing a Kelvin-type activation process. For almost pure carbonaceous particles only a fraction of $\leq 10^{-4}$ becomes activated to CCN. Increasing the coating of the particles with 
water-soluble $\mathrm{H}_{2} \mathrm{SO}_{4}$ to $3 \%$ of the particle volume increases the potential $\mathrm{CCN}$ activation at a saturation ratio of 1.006 by about two orders of magnitude. The presence of nucleated $\mathrm{H}_{2} \mathrm{SO}_{4}-\mathrm{H}_{2} \mathrm{O}$ particles enhances the mass transfer of water soluble sulphuric acid from the gas phase to the surface of the combustion particles by particle coagulation, which results in a further reduction of the $\mathrm{CCN}$ activation diameter. On the other hand, a high fraction of non-volatile organic matter in the particles causes a considerable increase in the activation diameter. However, the reduction of the $\mathrm{CCN}$ activation potential of the combustion particles by the non-volatile OC fraction can balance the increase in the potential activation by coating with $\mathrm{H}_{2} \mathrm{SO}_{4}$ only to some extent. Although the data are obtained from particles emitted from a gas turbine combustor, the competing effects of OC reducing $\mathrm{CCN}$ activation and coating with water-soluble matter increasing $\mathrm{CCN}$ activation are considered important also for combustion particles emitted from other sources.

The presented analysis is far from being quantitative, and the experimental data is limited. However, it presents important experimental material for the discussion on how organic components influence the $\mathrm{CCN}$ activity of internally mixed particles. Research on this subject focused so far on the role of organic acids and water-soluble organic matter. The experiments showed that soluble organic compounds activate according to Köhler theory whereas highly insoluble species do not activate at atmospherically relevant supersaturations (e.g., Novakov and Corrigan, 1996; Giebl et al., 2002; Broekhuizen et al., 2004). Comparing CCN activation diameters of organic acids at $\mathrm{S}_{w}=1.005$ (Corrigan and Novakov, 1999) with the values reported here shows, that even less active organic acids like adipic acid exhibit $\mathrm{D}_{\mathrm{CCN}}$ values of $116 \mathrm{~nm}$, while even coated combustion particles activate only for sizes larger than $\mathrm{D}_{\mathrm{CCN}} \cong 140 \mathrm{~nm}$.

For an urban aerosol which likely contains larger fractions of combustion particles, Saxena and co-workers (1995) reported that organic compounds diminish water adsorption of the inorganic fraction by about $30 \%$, while for non-urban aerosol the organic fraction increases the water adsorption. The PartEmis results indicate an effect of the organic fraction on the CCN activation potential similar to urban aerosol which contains a considerable fraction of combustion particles. Furthermore, the CCN activation of pure and coated carbon black particles was recently investigated using particles which were produced by nebulising an aqueous suspension of carbon black in a collision atomizer (Dusek et al., 2005). The activation of pure carbon black particles was found to require higher saturation ratios than predicted by Kelvin theory for insoluble, wettable spheres. Theoretical calculations indicated that the deviation of measured activation from Kelvin theory was consistent with a non-zero contact angle of the order of 4-6 deg of water at the soot surface. Coating the soot particles with a small amount of $\mathrm{NaCl}$ by adding $5 \%$ by mass $\mathrm{NaCl}$ to the carbon black suspension greatly enhanced their $\mathrm{CCN}$ efficiency. The measured

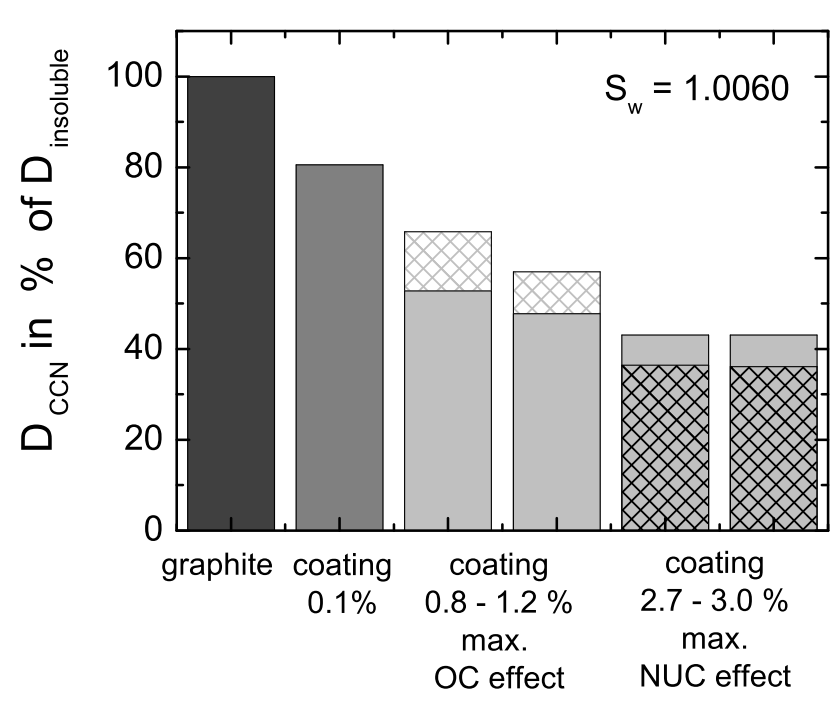

Fig. 15. Relative contribution of particle coating with sulphuric acid to the change in the $\mathrm{CCN}$ activation diameter of an initially insoluble graphite particle; the shaded bars represent the increase in $\mathrm{D}_{\mathrm{CCN}}$ and thus the reduction in $\mathrm{CCN}$ activation by a large fraction of non-volatile OC (OC effect) and the further decrease in $\mathrm{D}_{\mathrm{CCN}}$ and thus the enhancement of $\mathrm{CCN}$ activation by particle nucleationcoagulation (NUC effect).

CCN efficiencies were consistent with Köhler theory for particles consisting of insoluble and hygroscopic material. The PartEmis results for combustion particles point in the same direction as the observations for urban aerosol particles and carbonaceous particles generated in the laboratory.

The effect of particle ageing in the atmosphere which tends to increase the $\mathrm{CCN}$ activation potential due to oxidation of the particle surface was investigated by Hallett and coworkers (1989) for particles emitted from kerosene flames. $24 \mathrm{~h}$ of ageing increased the ratio $\mathrm{CCN} / \mathrm{N}$ only from $<2 \%$ to $3 \%$. Similar investigations of the influence of particle ageing on the CCN activation potential of combustion particles were not performed during PartEmis. Translating the ageing results from Hallet et al. to the PartEmis aerosol suggests that by far the major contribution to the $\mathrm{CCN}$ activation potential of combustion particles can be assigned to the coating with water-soluble matter which increases the ratio $\mathrm{CCN} / \mathrm{CN}$ by two orders of magnitude at $\mathrm{S}_{w}=1.006$. Broekhuizen and coworkers (2005) state in agreement with our observations, that solubility appears to be the driving force behind $\mathrm{CCN}$ activation for urban or combustion aerosols, respectively. However, the work by Dusek et al. (2005) indicates also an effect of the surface tension of the liquid layer forming on a combustion particle on its $\mathrm{CCN}$ activation potential. McFiggans et al. (2005) describe in their comprehensive discussion of the effects of physical and chemical aerosol properties on warm cloud droplet activation the variety of processes being relevant. The present paper adds comparable data on aerosol microphysics, aerosol chemistry, and $\mathrm{CCN}$ activation to this 
picture. However, more experimental data are required for solving the important problem of CCN activation of aerosol particles.

Acknowledgements. The PartEmis project was funded by the European Commission and by the Swiss Bundesamt für Bildung und Wissenschaft under contracts no. G4RD-CT-2000-00207 and 99.0632, respectively. The authors are very grateful to C. W. Wilson and the test rig operation crew at QinetiQ for their very strong support during the experiments. Valuable contributions from C. Stein and L. Fritzsche (DLR), as well as from H. Giebl (University of Vienna) during the analysis of the PartEmis data set are gratefully acknowledged.

Edited by: U. Pöschl

\section{References}

Broekhuizen, K., Pradeep Kumar, P., and Abbatt, J. P. D.: Partially soluble organics as cloud condensation nuclei: Role of trace soluble and surface active species, Geophys. Res. Lett., 31, L01107, doi:10.1029/2003GL018203, 2004.

Broekhuizen, K., Chang, R. Y.-W., Leatch, W. R., Li, S.-M., and Abbatt, J. P. D.: Closure between measured and modelled cloud condensation nuclei (CCN) using size-resolved aerosol compositions in downtown Toronto, Atmos. Chem. Phys. Discuss., 5, 6263-6293, 2005,

SRef-ID: 1680-7375/acpd/2005-5-6263.

Cachier, H., Bremond, M. P., and Biat-Ménard, P.: Determination of atmospheric soot carbon with a simple thermal method, Tellus, 41B, 379-390, 1989.

Charslon, R. J., Seinfeld, J. H., Nenes, A., Kulmala, M., Laaksonen, A., and Facchini, M. C.: Reshaping the theory of cloud formation, Science, 292, 2025-2026, 2001.

Chughtai, A. R., Miller, N. J., Smith, D. M., and Pitts, J. R.: Carbonaceous particle hydration III, J. Atmos. Chem., 34, 259-279, 1999.

Corrigan, C. E. and Novakov, T.: Cloud condensation nucleus activity of organic compounds: a laboratory study, Atmos. Environ., 33, 2661-2668, 1999.

Dusek, U., Ctyroky, P., Reischl, G., and Hitzenberger, R.: CCN activation of pure and coated carbon black particles, Environ. Sci. Technol., accepted, 2005.

Facchini, M. C., Mircea, M., Fuzzi, S., and Charlson, R. J.: Cloud albedo enhancement by surface-active organic solutes in growing droplets, Nature, 401, 257-259, 1999.

Giebl, H., Berner, A., Reischl, G., Puxbaum, H., and Hitzenberger, R.: CCN activation of oxalic acid and malonic acid test aerosols with the Univ. of Vienna cloud condensation nuclei counter, J. Aerosol Sci., 33, 1623-1634, 2002.

Gysel, M., Weingartner, E., and Baltensperger, U.: Hygroscopicity of Aerosol particles at low temperatures: 2. Theoretical and experimental hygroscopic properties of laboratory generated aerosols, Environ. Sci. Technol., 36, 63-68, 2002.

Gysel, M., Nyeki, S., Weingartner, E., Baltensperger, U., Giebl, H., Hitzenberger, R., Petzold, A., and Wilson, C. W.: Properties of jet engine combustor particles during the PartEmis experiment. Hygroscopicity at subsaturated conditions, Geophys. Res. Lett., 30, 1566, doi:10.1029/2003GL016896, 2003.
Hallet, J., Hudson, J. G., and Rogers, C. F.: Characterization of combustion aerosols for haze and cloud formation, Aerosol Sci. Technol., 10, 70-83, 1989.

Hitzenberger, R., Giebl, H. , Petzold, A., Gysel, M., Nyeki, S., Weingartner, E., Baltensperger, U., and Wilson, C. W.: Properties of jet engine combustor particles during the PartEmis experiment. Hygroscopic properties at supersaturated conditions, Geophys. Res. Lett., 30, 1779, doi:10.1029/2003GL017294, 2003.

Katragkou, E., Wilhelm, S., Arnold, F., and Wilson, C. W.: First gaseous Sulfur (VI) measurements in the simulated internal flow of an aircraft gas turbine engine during project PartEmis, Geophys. Res. Lett., 31, 2117, doi:10.1029/2003GL018231, 2004.

Kulmala, M., Vesala, T., and Kalkinnen, J.: Data for phase transitions in aerosol systems Manuscript for laboratory use, University of Helsinki, Helsinki, 1997.

Kumar, P. P., Broekhuizen, K., and Abbatt, J. P. D.: Organic acids as cloud condensation nuclei: Laboratory studies of highly soluble and insoluble species, Atmos. Chem. Phys., 3, 509-520, 2003, SRef-ID: 1680-7324/acp/2003-3-509.

Lammel, G. and Novako, T.: Water nucleation properties of carbon black and diesel soot particles, Atmos. Environ., 29, 813-823, 1995.

Lohmann, U. and Feichter J.: Global indirect aerosol effects: a review, Atmos. Chem. Phys., 5, 715-737, 2005,

SRef-ID: 1680-7324/acp/2005-5-715.

McFiggans, G., Artaxo, P., Baltensperger, U., et al.: The effect of physical and chemical aerosol properties on warm cloud droplet activation, Atmos. Chem. Phys. Discuss., 5, 8507-8646, 2005, SRef-ID: 1680-7375/acpd/2005-5-8507.

Novakov, T. and Corrigan, C. E.: Cloud condensation nucleus activity of the organic component of biomass smoke particles, Geophys. Res. Lett., 23, 2141-2144, 1996.

Nyeki, S., Gysel, M., Weingartner, E., Baltensperger, U., Hitzenberger, R., Petzold, A., and Wilson, C. W.: Properties of jet engine combustion particles during the PartEmis experiment: Particle size spectra $(\mathrm{d}>15 \mathrm{~nm})$ and volatility, Geophys. Res. Lett., 31, L18105, doi:10.1029/2003GL020569, 2004.

Petzold, A., Ström, J., Ohlsson, S., and Schröder, F. P.: Elemental composition and morphology of ice crystal residual particles in cirrus clouds and contrails, Atmos. Res., 49, 21-34, 1998.

Petzold, A., Stein, C., Nyeki, S., Gysel, M., Weingartner, E., Baltensperger, U., Giebl, H., Hitzenberger, R., Döpelheuer, A., Vrchoticky, S., Puxbaum, H., Johnson, M., Hurley, C. D., Marsh, R., and Wilson, C. W.: Properties of jet engine combustor particles during the PartEmis experiment. Microphysical and chemical properties, Geophys. Res. Lett., 30, 1719, doi:10.1029/2003GL017283, 2003.

Petzold, A. and Schönlinner, M.: Multi-angle absorption photometry - a new method for the measurement of aerosol light absorption and atmospheric black carbon, J. Aerosol Sci., 35, 421-441, 2004.

Petzold, A., Fiebig, M., Fritzsche, L., Stein, C., Schumann, U., Wilson, C. W., Hurley, C. D., Arnold, F., Katragkou, E., Baltensperger, U., Gysel, M., Nyeki, S., Hitzenberger, R., Giebl, H., Hughes, K. J., Kurtenbach, R., Wiesen, P., Madden, P., Puxbaum, H., Vrchoticky, S., and Wahl, C.: Particle emissions from aircraft engines - A survey of the European project PartEmis, Meteorol. Z., 14, 465-476, 2005.

Pitchford, M., Hudson, J. G., and Hallet, J.: Size and critical su- 
persaturation for condensation of jet engine exhaust particles, J. Geophys. Res., 96, 20 787-20 793, 1991.

Popovitcheva, O. B., Trukhin, M. E., Persiantseva, N. M., and Shonija, N. K.: Water adsorption on aircraft-combustor soot under young plume conditions, Atmos. Environ., 35, 1673-1676, 2001.

Pruppacher, H. R. and Klett, J. D.: Microphysics of Clouds and Precipitation, Kluwer 2nd edition, Academic Publishers, Dordrecht, 1997.

Puxbaum, H.: Thermo-Gasanalysator zur Charakterisierung von Kohlenstoff und Schwefelverbindungen in luftgetragenen Stäuben, Fresenius Z. Anal. Chem., 298, 250-259, 1979.

Saxena, P., Hildemann, L. M., McMurry, P. H., and Seinfeld, J. H.: Organics alter hygroscopic behaviour of atmospheric particles, J. Geophys. Res., 100, 18 755-18 770, 1995.

Schmid, H., Laskus, L., Abraham, H. J., Baltensperger, U., Lavanchy, V., Bizjak, M., Burba, P., Cachier, H., Crow, D., Chow, J., et al.: Results of the "carbon conference" international aerosol carbon round robin test stage I., Atmos. Environ., 35, 21112121,2001
Seisel, S., Lian, Y., Keil, T., Trukhin, M. E., and Zellner, R.: Kinetics of the interaction of water vapour with mineral dust and soot surfaces at T=298 K, Phys. Chem. Chem. Phys., 6, 1926-1932, 2004.

Vancassel, X., Sorokin, A., Mirabel, P., Petzold, A., and Wilson, C. W.: Volatile particles formation during PartEmis: a modelling study, Atmos. Chem. Phys., 4, 439-447, 2004,

SRef-ID: 1680-7324/acp/2004-4-439.

Weingartner, E., Burtscher, H., and Baltensperger, U.: Hygroscopic properties of carbon and diesel soot particles, Atmos. Environ. 31, 2311-2327, 1997.

Weingartner, E., Gysel, M., and Baltensperger, U.: Hygroscopicity of aerosol particles at low temperatures: 1 . New low-temperature H-TDMA instrument: Setup and first applications, Environ. Sci Technol., 36, 55-62, 2002.

Wilson, C. W., Petzold, A., Nyeki, S., Schumann, U., and Zellner, R.: Measurement and Prediction of Emissions of Aerosols and Gaseous Precursors from Gas Turbine Engines (PartEmis): An Overview, Aerospace Sci. Technol., 8, 131-143, 2004. 